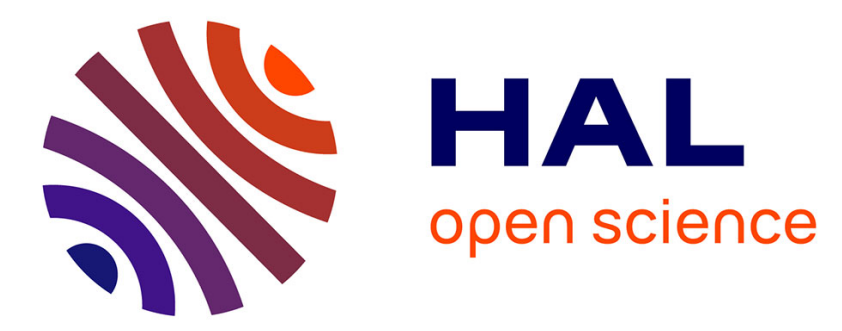

\title{
Periodic orbits, damped transitions and targeted energy transfers in oscillators with vibro-impact attachments
}

Young Lee, Francesco Nucera, Alexander Vakakis, Michael Mcfarland, Lawrence A Bergman

\section{- To cite this version:}

Young Lee, Francesco Nucera, Alexander Vakakis, Michael Mcfarland, Lawrence A Bergman. Periodic orbits, damped transitions and targeted energy transfers in oscillators with vibro-impact attachments. Physica D: Nonlinear Phenomena, 2009, 238, pp.1868 - 1896. 10.1016/j.physd.2009.06.013 . hal01510829

\section{HAL Id: hal-01510829 \\ https://hal.science/hal-01510829}

Submitted on 20 Apr 2017

HAL is a multi-disciplinary open access archive for the deposit and dissemination of scientific research documents, whether they are published or not. The documents may come from teaching and research institutions in France or abroad, or from public or private research centers.
L'archive ouverte pluridisciplinaire HAL, est destinée au dépôt et à la diffusion de documents scientifiques de niveau recherche, publiés ou non, émanant des établissements d'enseignement et de recherche français ou étrangers, des laboratoires publics ou privés. 


\title{
Periodic orbits, damped transitions and targeted energy transfers in oscillators with vibro-impact attachments
}

\author{
Young S. Lee ${ }^{\mathrm{a}}$, Francesco Nucera ${ }^{\mathrm{b}}$, Alexander F. Vakakis ${ }^{\mathrm{c}}$, D. Michael McFarland ${ }^{\mathrm{d}}$, \\ Lawrence A. Bergman ${ }^{\mathrm{d}}$ \\ ${ }^{a}$ Department of Mechanical and Aerospace Engineering, New Mexico State University, Las Cruces, NM 88003, USA \\ ${ }^{\mathrm{b}}$ Department of Mechanics and Materials, Mediterranean University of Reggio Calabria, 89122 Reggio Calabria, Italy \\ ${ }^{c}$ Department of Mechanical Science and Engineering, University of Illinois at Urbana-Champaign, Urbana, IL 61801, USA \\ d Department of Aerospace Engineering, University of Illinois at Urbana-Champaign, Urbana, IL 61801, USA
}

\begin{abstract}
We study complex damped and undamped dynamics and targeted energy transfers (TETs) in systems of coupled oscillators, consisting of single-degree-of-freedom primary linear oscillators (LOs) with vibroimpact attachments, acting, in essence, as vibro-impact nonlinear energy sinks (VI NESs). First, the complicated dynamics of such VI systems is demonstrated by computing the VI periodic orbits of underlying Hamiltonian systems and depicting them in appropriate frequency-energy plots (FEPs). Then, VI damped transitions and distinct ways of passive TETs from the linear oscillators to the VI attachments for various parameter ranges and initial conditions are investigated. As in the case of smooth stiffness nonlinearity [Y. Lee, G. Kerschen, A. Vakakis, P. Panagopoulos, L. Bergman, D.M. McFarland, Complicated dynamics of a linear oscillator with a light, essentially nonlinear attachment, Physica D 204 (1-2) (2005) 41-69], both fundamental and subharmonic TET can be realized in the VI systems under consideration. It is found that the most efficient mechanism for VI TET is through the excitation of highly energetic VI impulsive orbits (IOs), i.e., of periodic or quasiperiodic orbits corresponding to zero initial conditions except for the initial velocities of the linear oscillators. In contrast to NESs with smooth essential nonlinearities considered in previous works, VI NESs are capable of passively absorbing and locally dissipating significant portions of the energies of the primary systems to which they are attached, at fast time scale. This renders such devices suitable for applications, like seismic mitigation, where dissipation of vibration energy in the early, highly energetic regime of the motion is a critical requirement.
\end{abstract}

Keywords:

Targeted energy transfer (TET)

Vibro-impact nonlinear energy sink (VINES)

Passive and broadband vibration absorber

\section{Introduction}

\subsection{Background}

Passive, broadband targeted energy transfer (TET) refers to the one-way directed transfer of energy from a primary subsystem to a nonlinear attachment; this phenomenon is realized in damped, coupled, essentially nonlinear oscillators through resonance captures and escapes along intrinsic periodic or quasiperiodic orbits of the underlying Hamiltonian systems [1,2]. Indeed, TET is realized from primary linear or nonlinear systems to essentially nonlinear attachments, acting as nonlinear energy sinks (NESs). An NES generally requires two elements: an essentially nonlinear (i.e., nonlinearizable) stiffness and a (usually, linear viscous) damper. The former, which is smooth in many cases, enables the NES to resonate with any of the linearized modes of the primary system to which it is attached, whereas the latter acts as dissipator of the vibrational energy transferred through resonant modal interactions (see [3,4] for an overview of the dynamics governing TET and its various applications). In most previous works smooth essential (purely cubic) stiffness nonlinearities were considered, although in some recent works nonsmooth stiffness nonlinearities were studied as well [5-10].

Indeed, NESs with clearances and vibro-impacts have been considered in applications where vibration reduction at a fast time scale is required. The consequence of the capacity of this class of nonsmooth NESs for rapid energy absorption and dissipation makes them applicable to applications where the energy transfer from the directly forced primary structure to the NES(s) must be accomplished at the early stage of the motion, if possible immediately after the application of an external shock. Examples 
include structures under seismic excitation [5-7] and cars during collision.

The regular and chaotic dynamics and bifurcations of vibroimpact (VI) oscillators have been studied extensively in the literature [11-16]. In a series of papers [17-24], VI dampers were considered for reducing the vibration levels of structures under periodic or stochastic excitation. Shaw and Holmes [25], Shaw and Shaw [26] and Shaw [27] applied methods from the geometrical theory of nonlinear dynamics to analyze the dynamics of free and forced dynamics of systems with piecewise nonlinearities, including systems with vibro-impact nonlinearities. Recently, Gorelyshev and Neishtadt [28] discussed the extension of adiabatic perturbation theory to VI systems; Mikhlin et al. [29] and others [30-37] studied periodic orbits, bifurcations and chaos in discrete and continuous oscillators with clearance nonlinearities or vibroimpacts; Zhuravlev $[38,39]$ investigated vibro-impact oscillations using nonsmooth coordinate transformations (for an additional application of this method see also [40]), and Pilipchuk [41] extended this approach by considering nonsmooth transformations of the dependent (temporal) variable of the problem.

Pinnington [42] analyzed energy exchange and dissipation due to collisions in a line of coupled oscillators, and Salapaka et al. [43] studied the dynamics of a linear oscillator impacting with a vibrating platform. Quinn [44] investigated the oscillations of two parametrically excited pendula undergoing vibro-impacts; and Li and Darby [45] reported experimental work on the effect of an impact damper on an MDOF system. Murphy and Morrison [46] studied, computationally and experimentally, instabilities and bifurcations of a vibro-impacting string; and $\mathrm{Hu}$ and Schiehlen [47] discussed multi-scale simulation of impact responses with applications from wave propagation to rigid body dynamics. Sampaio and Soize [48] formulated measures that quantify nonlinear effects for uncertain systems, whereas Azeez and Vakakis [49] approached the issue of nonlinear effects quantification using proper orthogonal decomposition. Vedenova et al. [50] and others [51,52] examined modeling inelastic impacts with smooth, essentially nonlinear functions of high degree; and Nayeri et al. [53] investigated the action of multi-unit impact dampers in systems under stochastic excitation. Namachchivaya and Park [54] developed an analytical approach based on averaging for studying the dynamics of VI systems under stochastic excitation; Wagg [55] used energy balance analysis to examine multi-modal systems undergoing vibro-impacts, and studied effective restitution coefficients; and Shaw and Pierre [56] applied tuned impact dampers in rotating structures and assessed their performance.

In the works by Georgiades et al. [8,9] TETs in coupled mechanical oscillators with NESs possessing nonsmooth stiffnesses (referred to from now on as nonsmooth NESs or NS NESs) were analyzed by studying the shock isolation properties of systems of coupled nonconservative linear oscillators with NESs possessing clearance nonlinearities (i.e., piecewise linear oscillators). Apart from the fact that such nonsmooth stiffness elements introduce strong nonlinearities to the systems to which they are attached, they are rather easy to implement in practical settings as combinations of linear stiffnesses. Numerical evidence of the capacity for shock isolation of NS NESs was provided in these works; that is, significant energy transfers from the primary systems to such NESs can occur right from the beginning of the motion (i.e., during the energetically high regime of the dynamics). The capacity of NS NESs to rapidly absorb shock energy in the initial highly energetic regime is critical to their role as shock isolators.

Karayannis et al. [10] then extended this study to the case where a primary system experiences an impulsive excitation, with the nonlinear attachment undergoing two-sided inelastic impacts. Superior shock absorption by the VI NES compared to the linear absorber is attained for intermediate clearances (i.e., away from
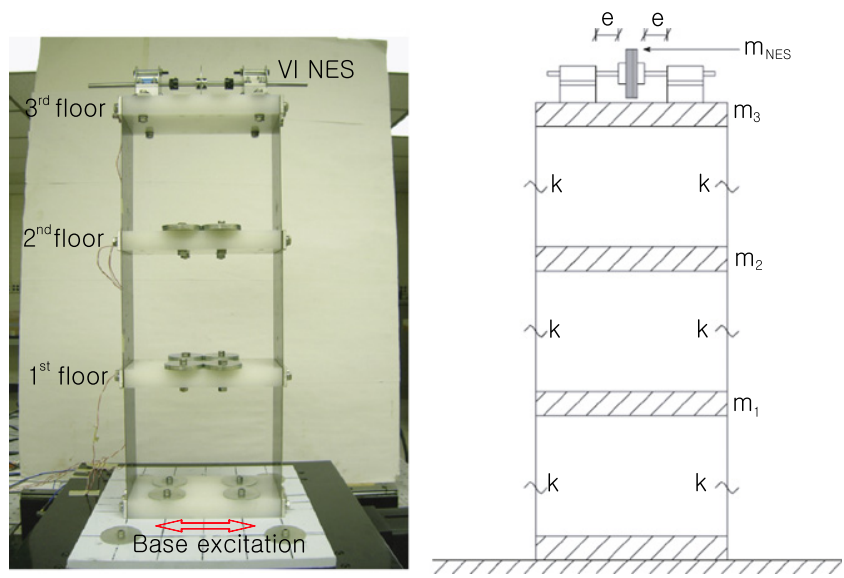

Fig. 1. Experimental setup of a 3-story building with a VI NES installed at its top floor [5].

the two limiting linear systems corresponding to zero and large clearances, respectively), small coupling frequencies, and large mass ratios. Moreover, high shock absorption efficiencies was attained for even a small total number of vibro-impacts, provided that conditions for sufficient momentum and energy exchanges between the primary system and the NES during vibro-impacts were realized. It was also shown that VI NESs can be designed as effective shock isolators, providing significant reduction of maximum responses of the primary systems over broad frequency ranges. Hence, appropriately designed VI NESs can act as broadband, passive shock isolators. The results indicated that, in designing VI NESs as shock isolators, important design parameters are the clearances, the coupling stiffnesses and the NES masses. Moreover, better vibro-impact shock absorption is anticipated for weak coupling stiffness between the primary systems and the attached NESs, and relatively large values of NES masses compared to the masses of the corresponding primary systems. These results are in agreement with findings of Georgiades et al. [8].

\subsection{Motivations for the present study}

In previous works, application of VI NESs for passive seismic mitigation was studied theoretically and experimentally in [5-7] - e.g., Fig. 1, where an experimental model of a 3-story building with an attached VI NES under base excitation is depicted. In particular, Nucera et al. [5] considered a single-degree-of-freedom linear oscillator (LO) with no viscous damping coupled to a VI NES as a simplified model for the fixture of Fig. 1. Fig. 2(a) depicts a VI damped transition for this system, from which we deduce that there exist three distinct regimes for this VI damped transition. Moreover, the instantaneous energy plot and wavelet transform spectra of the relative displacement between the linear oscillator and the VI NES depicted in Fig. 2(b) indicate that there occur multi-frequency resonance captures $[57,58]$ in the dynamics as the energy diminishes due to energy dissipation at the impact instant. Clearly, there is the need for developing techniques capable of analyzing and identifying such complex VI transitions, which will enable us to categorize and classify different types of multifrequency resonance captures that occur in VI orbits.

In an attempt to address these issues, in this work we will introduce some new tools that will help us understand, classify and systematically study complex transitions in the simplest possible VI system, namely, a linear oscillator with a VI attachment. Although these tools will be applied exclusively to this two-DOF system, they are rather general so they can be applied to higher-dimensional VI systems as well. First, we will focus on the detailed computation of 

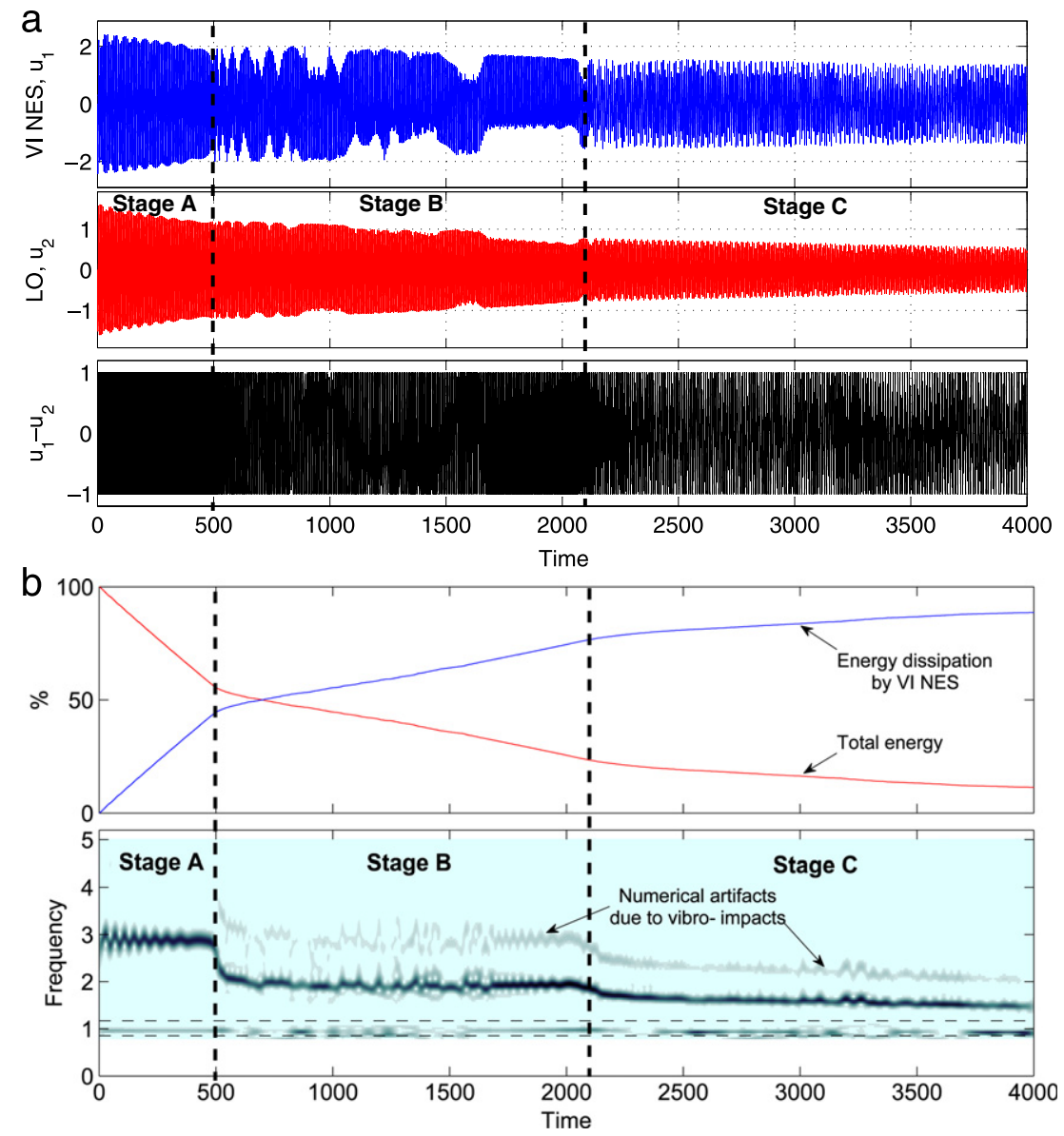

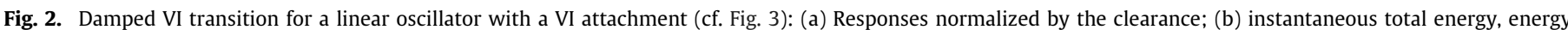
dissipation due to vibro-impacts, and wavelet transform (WT) spectra of the relative displacement between the linear oscillator and the VI NES.

the VI periodic solutions of the underlying Hamiltonian system and on their depiction on a frequency-energy plot (FEP). The rich structure of periodic orbits (and impulsive orbits) of the VI system then will become immediately apparent. In the sequence we will study damped transitions of the VI system undergoing inelastic vibroimpacts, by superimposing the WT spectra of transient VI damped responses on the Hamiltonian FEP. This will allow us to detect and understand complex nonlinear modal interactions occurring during the VI transitions; in the process we will study the different dynamical mechanisms that govern VI targeted energy transfer (TET) in the two-DOF system; by doing so we will generalize previous results on TET in dynamical systems with strong but smooth stiffness nonlinearities. Our study will demonstrate schematically the complex resonance captures and multi-modal interactions occurring during these VI transitions. In addition, we will provide a numerical study of VI TET efficiency by considering the excitation of the two-DOF system by external impulses, and end with some concluding remarks.

\section{Hamiltonian system with elastic vibro-impacts: Periodic orbits in the frequency-energy plot (FEP)}

To study the underlying dynamical mechanisms and associated transient resonance captures (TRCs) that govern passive TET in systems with VI NESs, and also to demonstrate the complexity that a single VI NES can induce in the dynamics, we consider the simplest primary system - VI NES configuration, namely a single-degree-of-freedom (SDOF) LO coupled to a VI NES (Fig. 3). As in the case of the NES with smooth essential nonlinearities studied in previous works, we will show that a clear interpretation

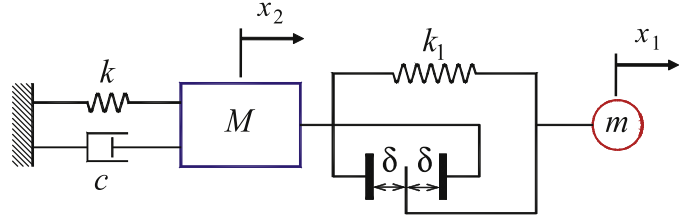

Fig. 3. An SDOF linear oscillator coupled to a VI NES.

of damped VI transitions that govern TET in the shock-excited system can be gained by depicting the WT spectra of these motions on an FEP of the underlying VI Hamiltonian system (corresponding to the system with purely elastic impacts and no viscous damping elements). Also, we will demonstrate that, for sufficiently weak dissipation, weakly damped VI transitions take place near branches of periodic or quasiperiodic motions of the corresponding undamped system. Hence, by studying the structure of periodic orbits of the Hamiltonian system, we should be able to understand the governing dynamics of the weakly damped dynamics as well, and to clearly identify complex multi-frequency transitions and resonance captures leading to energy exchanges and TET in the weakly damped VI system.

We initiate our study by computing the periodic orbits of the Hamiltonian system with no viscous damping dissipation and purely elastic vibro-impacts and depicting them in a frequencyenergy plot(FEP). Considering the two-degrees-of-freedom system of Fig. 3, we note that the equations of motion are linear in time intervals between vibro-impacts (i.e., $\left|x_{2}-x_{1}\right|<\delta$ ), and can be written as

$m \ddot{x}_{1}+k_{1}\left(x_{1}-x_{2}\right)=0$

$M \ddot{x}_{2}+c \dot{x}_{2}+k x_{2}+k_{1}\left(x_{2}-x_{1}\right)=0$ 
or in non-dimensional form,

$\mu \ddot{u}_{1}+\sigma\left(u_{1}-u_{2}\right)=0$

$\ddot{u}_{2}+\lambda \dot{u}_{2}+u_{2}+\sigma\left(u_{2}-u_{1}\right)=0$.

Dots in (2) denote differentiation with respect to the transformed temporal variable $\xi=\sqrt{k / M} t$; moreover, $\mu=m / M$, denotes the mass ratio; $\sigma=k_{1} / k$, the stiffness ratio; and $\lambda=c / \sqrt{M k}$, the damping factor. In addition, in Eq. (2) we consider normalized displacements through the rescalings $u_{i}=x_{i} / \delta, i=1,2, \delta \neq 0$, so in terms of these normalized displacements vibro-impacts occur when $\left|u_{2}-u_{1}\right|=1$. Note that the mass of the NES is not necessarily small compared to that of the LO, so the ratio $\mu$ in Eq. (2) is assumed to be an $\mathcal{O}(1)$ quantity [10].

Assuming zero viscous damping (i.e., $\lambda=0$ ) and considering purely elastic impacts (i.e., with restitution coefficient $\rho=1$ ), the VI system of Fig. 3 becomes Hamiltonian. The velocities of the LO and the NES after an impact (denoted by superscripts '+') are computed in terms of the corresponding velocities before impact (denoted by superscripts '-') by the following relations:

$\dot{u}_{1}^{+}=\frac{(\mu-1) \dot{u}_{1}^{-}+2 \dot{u}_{2}^{-}}{1+\mu}, \quad \dot{u}_{2}^{+}=\frac{2 \mu \dot{u}_{1}^{-}+(1-\mu) \dot{u}_{2}^{-}}{1+\mu}$.

In the numerical algorithm used to compute the VI responses, the linear equations (2) are integrated for as long as the no-impact condition $\left|u_{2}-u_{1}\right|<1$ is satisfied. When $\left|u_{2}-u_{1}\right|=1$, a (purely elastic) impact occurs, and discontinuities in the velocities take place, whereas the displacements remain continuous throughout the impact. The velocities immediately after the impact are computed by relations (3), and the numerical integration of the linear system (2) resumes with the new initial conditions until the next impact occurs, where this procedure is iterated. As discussed in earlier works $[5,10]$, precise computation of the time instants where vibro-impacts occur is essential for the accuracy of the numerical simulations. This accuracy was checked by recording the total energy of the VI motion and ensuring its conservation through the entire interval of the simulation. The total (conserved) normalized energy $H\left(u_{1}, \dot{u}_{1}, u_{2}, \dot{u}_{2}\right)$ is computed in terms of the initial conditions of the normalized system (2) as

$$
\begin{aligned}
H\left(u_{1}, \dot{u}_{1}, u_{2}, \dot{u}_{2}\right)= & \frac{1}{2}\left[\mu \dot{u}_{1}(0)^{2}+\dot{u}_{2}(0)^{2}\right] \\
& +\frac{1}{2}\left[u_{2}(0)^{2}+\sigma\left(u_{1}(0)-u_{2}(0)\right)^{2}\right]=h .
\end{aligned}
$$

Assuming zero initial velocities, the critical threshold for vibroimpacts to occur is computed as $h_{\min }=\sigma / 2$. Throughout this paper, the normalized mass and stiffness parameters are taken as $\mu=0.1$ and $\sigma=0.1$, respectively.

The periodic solutions of the Hamiltonian vibro-impacting system can be computed by employing the method of nonsmooth temporal transformations (NSTTs) first introduced by Pilipchuk [41] and Pilipchuk et al. [59]. NSTTs were applied in [57] for computing the periodic orbits (or nonlinear normal modes; NNMs [60]) of the corresponding system with smooth essential nonlinearity (i.e., with a 'smooth' NES). To this end, we introduce the coordinate transformation

$u_{i}(\xi)=e(\xi / \alpha) y_{i}(\tau(\xi / \alpha)), \quad i=1,2$

where $\alpha=T / 4$ represents the quarter-period of the VI periodic motion, and the nonsmooth functions $\tau(\cdot)$ and $e(\cdot)$ are defined as (see also Fig. 4)

$\tau(x)=\frac{2}{\pi} \sin ^{-1}\left(\sin \frac{\pi}{2} x\right), \quad e(x)=\frac{\mathrm{d}}{\mathrm{d} x} \tau(x)$.

Then, we obtain the two-point nonlinear boundary value problem (NLBVP) in terms of the variables $y_{1}, y_{2}, y_{3}=y_{1}^{\prime}$ and $y_{4}=y_{2}^{\prime}$
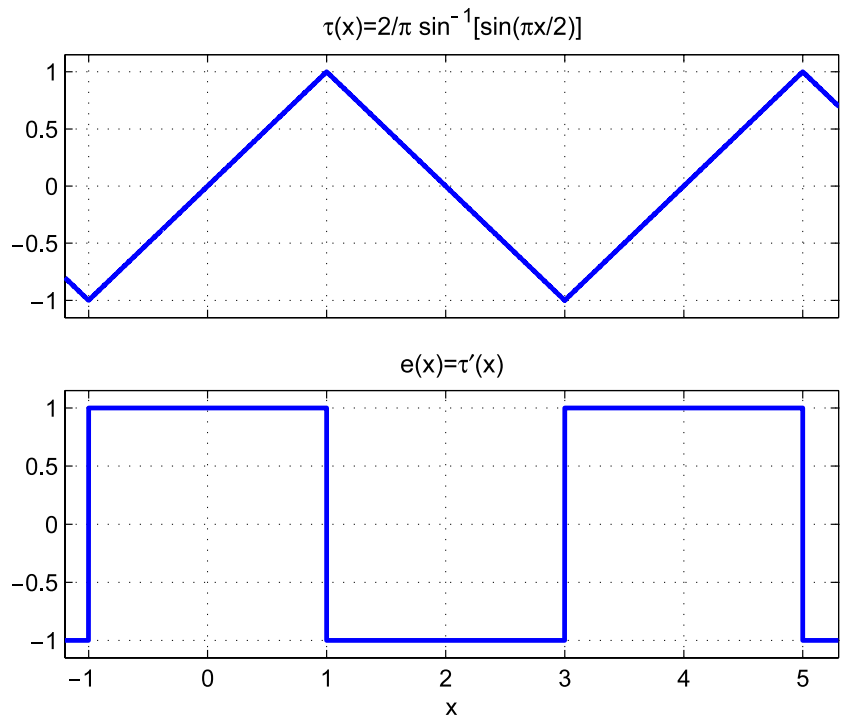

Fig. 4. The two nonsmooth basis functions, $\tau(x)$ and $e(x)=\mathrm{d} \tau(x) / \mathrm{d} x$.

$y_{1}^{\prime}=y_{3}$

$y_{2}^{\prime}=y_{4}$

$y_{3}^{\prime}=-\sigma \alpha^{2}\left(y_{1}-y_{2}\right) / \mu$

$y_{4}^{\prime}=-\alpha^{2} y_{2}-\sigma \alpha^{2}\left(y_{2}-y_{1}\right)$

$y_{1}( \pm 1)=y_{2}( \pm 1)=0$

where primes denote differentiations with respect to the nonsmooth variable $\tau$, and the periodic orbits are computed subject to zero initial velocities. Vibro-impacts occur when $\left|y_{2}-y_{1}\right|=1$, at which points discontinuities in the velocities through expressions similar to Eq. (3) are imposed. The solution of the NLBVP (7) computes the periodic orbit over half of its period $T=4 \alpha$ (i.e., for $-1 \leq \tau \leq 1 \Rightarrow-\alpha \leq \xi \leq \alpha)$; to extend the periodic orbit over the entire period we take into account the form of the nonsmooth transformations (5), such that the conserved energy of the periodic orbit is expressed as

$h=\frac{1}{2 \alpha^{2}}\left[\mu y_{1}^{\prime}(-1)^{2}+y_{2}^{\prime}(-1)^{2}\right]$.

The NLBVP (7) is solved by a shooting method (see, for example, [61-63]) in the bounded domain $-1 \leq \tau \leq 1$. However, unlike the shooting method employed for the analogous case of the NES with smooth nonlinearity in [57], matching at $\tau=0$ of the two solutions initiated from the left and right boundaries $(\tau= \pm 1)$ is not helpful in the current VI problem. This is due to the fact that symmetric VI periodic orbits are expected to exhibit vibroimpacts at $\tau=0$, so matching solutions at that point becomes meaningless. Therefore, in the VI problem we adopt the following alternative matching condition: For fixed quarter-period $\alpha$, the set of equations of the NLBVP is solved as an initial value problem with initial conditions at the left boundary, $y_{i}(-1)=0$ and $y_{i}^{\prime}(-1) \neq$ $0, i=1,2$. Then, perform matching at the right boundary through the inequalities, $\left|y_{i}\left(+1_{-}\right)\right|<\varepsilon \ll 1, i=1$, 2 , where the tolerance $\varepsilon$ is taken as $\mathcal{O}\left(10^{-5}\right)-\mathcal{O}\left(10^{-6}\right)$. This procedure ensures that the NLBVP (7) is approximately solved (that is, within the prescribed numerical tolerance).

It is anticipated that the seemingly simple VI system of Fig. 3 will possess a very complicated structure of periodic orbits in the FEP. This expectation is justified by the fact that vibroimpact nonlinearity represents a very strong (and degenerate) form of nonlinearity. Indeed, considering the family of essentially nonlinear stiffnesses

$f_{n}(u)=k_{n} u^{2 n+1}, \quad n=0,1, \ldots$, 


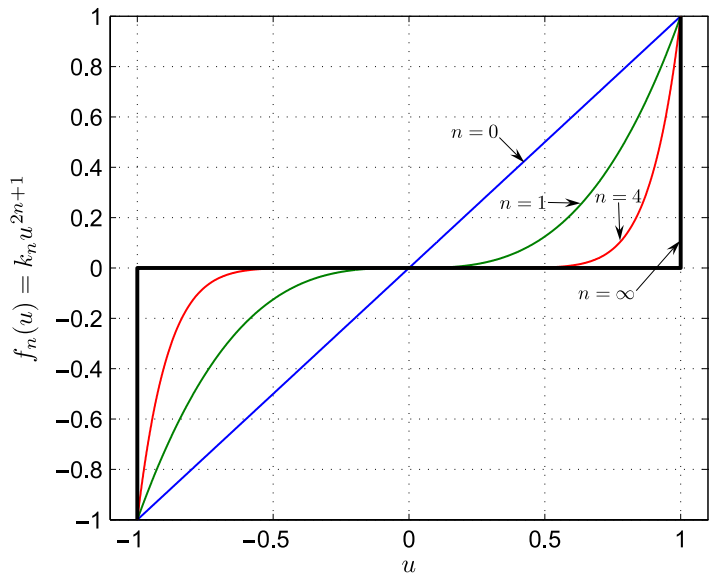

Fig. 5. Vibro-impact nonlinearity as limiting case of a family of smooth, essentially nonlinear stiffnesses.

the coefficient $k_{n}$ depends on the exponent $n$, and is selected so that the normalization condition $f_{n}( \pm 1)= \pm 1, \forall n$ is satisfied. Then, the vibro-impact nonlinearity corresponding to purely elastic impacts is obtained as the (degenerate) discontinuous limit $f_{\infty}(u)=\lim _{n \rightarrow \infty} f_{n}(u)$ (Fig. 5 ; [50,64]). Viewed in this context, vibro-impact nonlinearity can be considered as the 'strongest possible' stiffness nonlinearity of the family (9). Following this reasoning, Pilipchuk $[41,65,66]$ developed an asymptotic methodology based on nonsmooth transformations and nonsmooth generating functions that is applicable to strongly nonlinear regimes [59,67].

The anticipated high complexity of the structure of VI periodic orbits dictates the use of careful notation for their representation in the FEP. In principle, the basic notation introduced in [57] for the FEP of the dynamics of the Hamiltonian system with 'smooth' NES is followed, with an additional index introduced characterizing the pattern of the occurring vibro-impacts. To this end, we employ the following notation for depicting the various types of VI periodic orbits in the FEP:

Symmetric VI periodic orbits are denoted as $\operatorname{SmnE}(0) p p \pm$, and satisfy the conditions, $u_{i}(\xi)= \pm u_{i}(\xi+T / 2), \forall \xi \in \mathbb{R}, i=1,2$, where $T$ is the period of the motion. Similar to the 'smooth' NES [57], symmetric VI orbits correspond to synchronous oscillations of the LO and the VI NES, and typically are represented by curves in the configuration plane $\left(u_{1}, u_{2}\right)$ (cf. Fig. 29 in Appendix). Unsymmetric VI periodic orbits, labeled as Umnpq \pm , do not satisfy the conditions of the symmetric ones. These orbits correspond to asynchronous motions of the two oscillators and are represented by open or closed (Lissajous) curves in the configuration plane $\left(u_{1}, u_{2}\right)$ (cf. Fig. 30 in Appendix). The integer index $m$ denotes the number of half-waves in the VI NES response within a half-period, whereas the integer index $n$ denotes the corresponding number of half-waves in the LO response; clearly, the ratio m: $n$ indicates the order of nonlinear resonance that occurs between the VI NES and the LO for a given VI periodic orbit. Moreover, the index $E$ or $O$ denotes the symmetry pattern of the vibro-impacts, and has meaning only for symmetric VI orbits: $E(O)$ denotes an even (odd) symmetry of occurring vibro-impacts within a half-period; it follows that the notation $E(0)$ implies that a vibro-impact occurs (does not occur) at quarter-period $\xi=\alpha=T / 4$. The integer indices $p$ and $q$ denote the number of vibro-impacts that occur in the first and second quarter-period, respectively, of a given VI periodic orbit; it follows that for symmetric orbits $p=q$. Finally, the ' + ' sign corresponds to in-phase VI periodic motions, where, for zero initial displacements, the initial velocities of the LO and the NES possess identical signs at the beginning of both the first and second half-periods of the periodic motion. Otherwise, the VI periodic motion is deemed to be out-of-phase and the '-' sign is used. Finally, the two in-phase and out-of-phase linear modes of the system with zero clearance $(\delta=0)$ are denoted by $L m m \pm$ and are, in fact, equivalent to $L 11 \pm$.

The (incomplete) FEP of the Hamiltonian VI system for $\mu=$ $\sigma=0.1$ is depicted in Fig. 6, with some representative VI periodic orbits presented in Fig. 7 . The complexity of the bifurcations that generate the VI periodic orbits can be inferred from the bifurcation diagrams of Fig. 8 where the initial velocities of the LO and the VI NES (for zero initial displacements) are depicted as functions of the total energy $h$. As mentioned previously, this complexity was anticipated in view of the degenerate vibro-impact nonlinearity of this system.

Note that the FEP in Fig. 6 is obtained for system (2) with all displacements being normalized with respect to the clearance $\delta$,

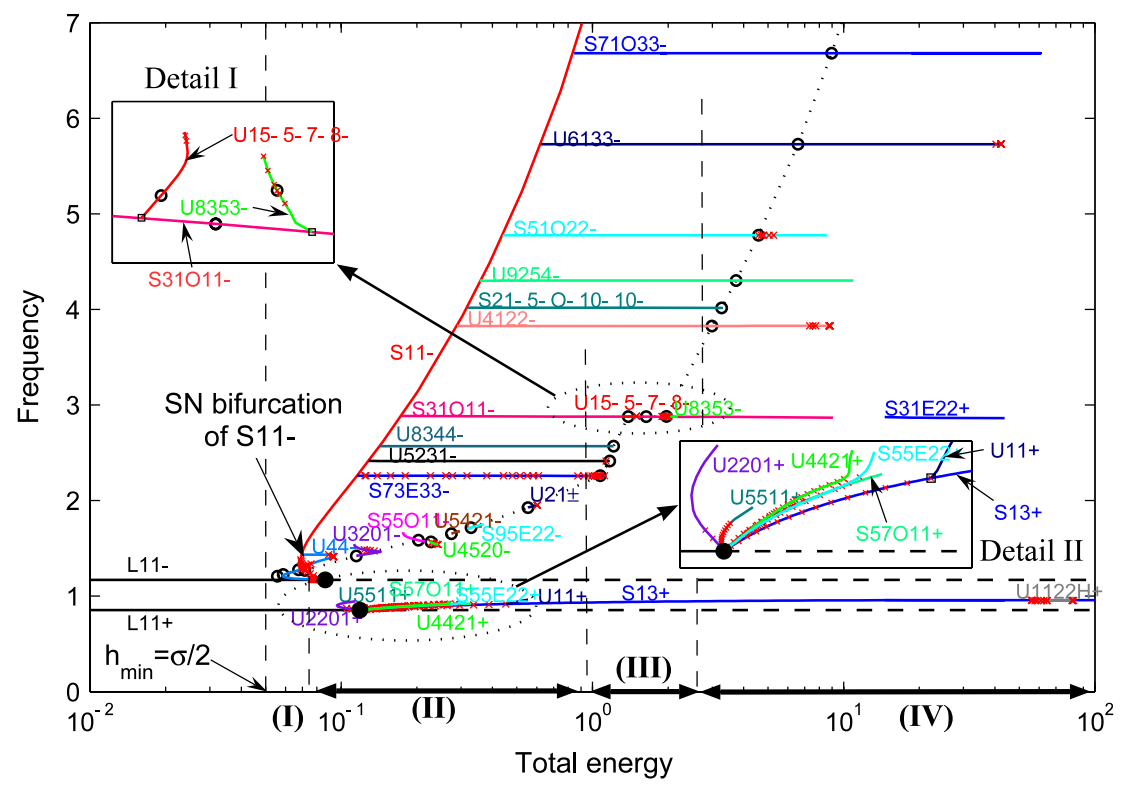

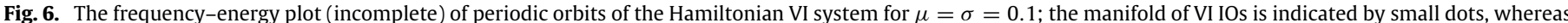
the thresholds for vibro-impacts for the in-phase and out-of-phase modes are denoted by bullets; unstable branches are denoted by crosses. 

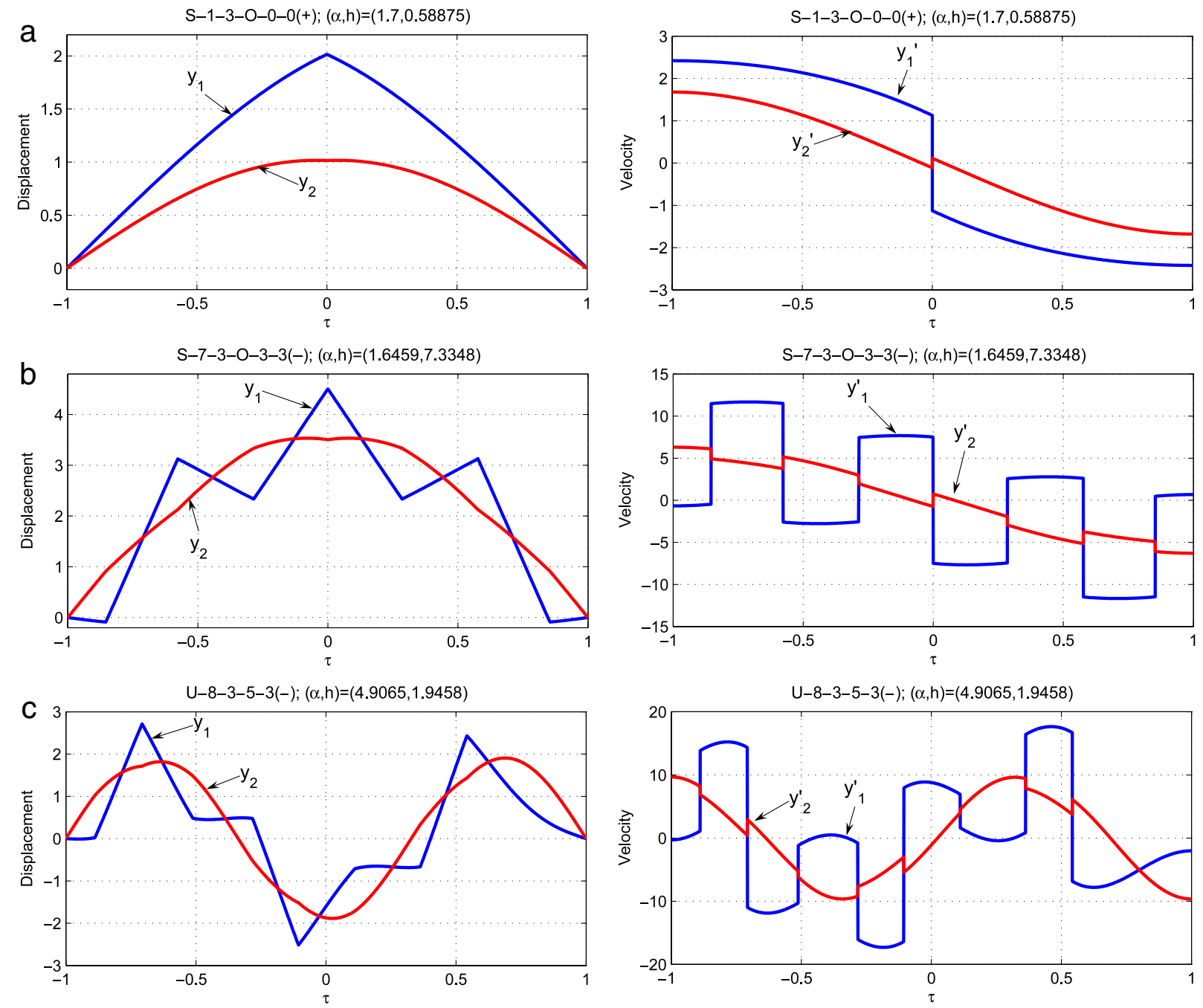

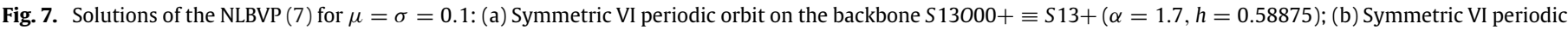
orbit $573033-(\alpha=1.6459, h=7.3348)$; (c) Unsymmetric VI periodic orbit $U 8853-(\alpha=4.9065, h=1.9458)$

so that vibro-impacts occur whenever the absolute value of the relative displacement between the two particles becomes equal to unity in magnitude. Considering the original system (1) with clearance $\delta$, its Hamiltonian is expressed as, $\hat{H}=\delta^{2} H$ where $H$ is the normalized Hamiltonian defined by (4). This implies that, for fixed system parameters, $\mu=0.1$ and $\sigma=0.1$, the Hamiltonian structure of the original (non-normalized) system will be identical to that of Fig. 6; it follows that for larger (smaller) clearances, the entire structure of VI periodic orbits will be preserved but just shifted towards higher (lower) energy regimes. So, the introduced normalization allows us to study all possible VI responses of the original system by considering a single 'normalized' FEP for fixed mass and coupling stiffness ratios. It is interesting to note that this normalization does not hold for the system with smooth stiffness nonlinearity (i.e., the 'smooth' NES); this can be easily deduced when noting that the introduced normalization changes the form of the system with smooth nonlinearities. Indeed, the topological structure of the FEP of the system with 'smooth' NES with pure cubic stiffness nonlinearity (and the corresponding bifurcation structure of the Hamiltonian periodic orbits) is affected by both the mass ratio and the essential stiffness nonlinearity of the NES [57].

We now make some comments and remarks regarding the 'normalized' FEP of Fig. 6. First, we note that the two bullets indicate the critical energy thresholds below which oscillations without vibro-impacts occur, and the dynamics of the two-DOF system is purely linear. Clearly, only the in-phase and out-of-phase linear normal modes $L 11 \pm$ exist below the energy thresholds. As we increase the energy of the motion above these energy thresholds, vibro-impacts start occurring, giving rise to two main branches of symmetric periodic VI NNMs: the branch of out-ofphase symmetric VI NNMs $S(2 m)(2 m) E m m-(m \geq 1$, integer) or $S(2 n+1)(2 n+1) O n n-(n \geq 0$, integer $)$, which bifurcates from the out-of-phase linear mode $L 11-$, after which this mode becomes unstable (cf. Fig. 28 in Appendix); and the branch of symmetric in-phase VI NNMs $S(2 m)(6 m) E m m+(m \geq 1)$ or $S(2 n+1)(6 n+$ $3)$ Onn+ $(n \geq 0)$ which bifurcates out of the in-phase linear mode $L 11+$, after which this linear mode also becomes unstable (cf. Fig. 27 in Appendix). For convenience, from hereon the shortened notations, $S 11-$ and $S 13+$, will be adopted for these two main backbone branches, which will be referred to as backbone (global) branches of the FEP. Both backbone branches exist over broad frequency and energy ranges and, except for the neighborhoods of the bifurcation points with $L 11 \pm$, they correspond to oscillations that are mainly localized to the VI NES. A basic bifurcation in the VI FEP is the saddle-node (SN) bifurcation of the backbone branch $S 11-$ (at $h \approx 0.06$ ), which signifies the elimination of the unstable branch $S 11-$ bifurcating out of $L 11-$; the stable branch S11- generated after this SN bifurcation maintains its stability for increasing energies. As shown below, this SN bifurcation of the backbone branch $S 11$ - significantly affects the capacity of the VI NES for TET. The additional in-phase backbone branch $\mathrm{S13}+$ 

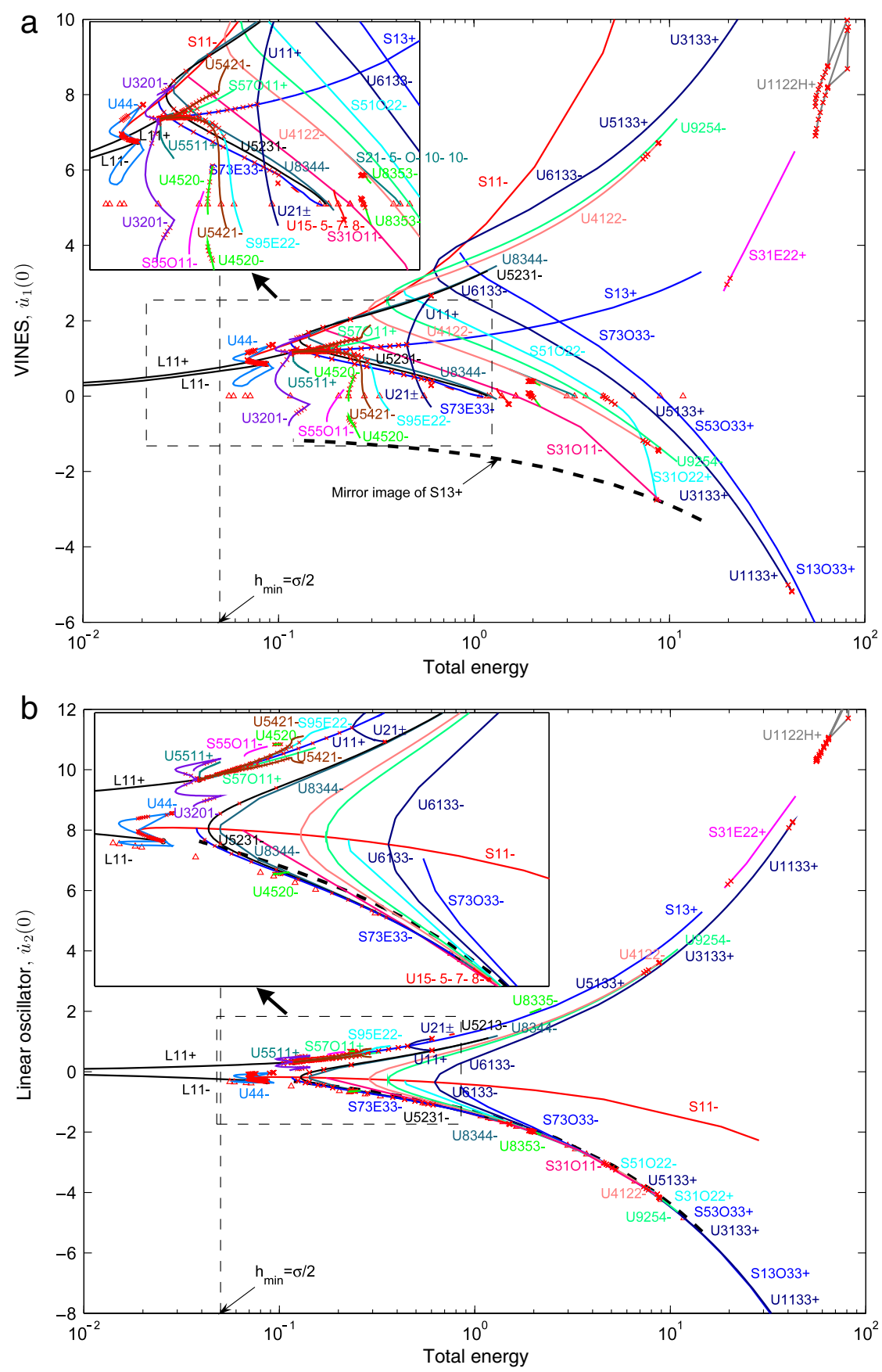

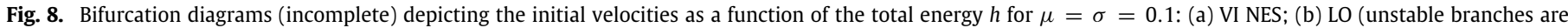
marked by crosses).

bifurcating out of the in-phase linear mode $L 11+$ is stable until high energies where zones of instability appear.

In Fig. 7(a) we depict a typical stable VI orbit lying on the inphase backbone branch $S 13+$. We note that, in the corresponding FEP of the system with 'smooth' NES studied in [57], there exist two backbone branches $S 11 \pm$. As indicated by the time series of Fig. 7(a), however, on the in-phase VI backbone branch three sign changes for the LO velocity within half a period are realized, compared to only one for the NES velocity; this high-frequency component becomes more prominent at higher energies (in addition, as will be shown in Section 3.1, this explains the 3:1 resonant modal interactions observed in wavelet transform spectra when the dynamics is initiated on branch $S 13+$ for weak damping).

A different class of VI periodic solutions of the FEP lies on subharmonic tongues (local branches); these are multi-frequency periodic motions, possessing frequencies that are rational multiples of one of the linearized eigenfrequencies of the system. Similar to the FEP of the system with 'smooth' NES [57], each subharmonic tongue is defined over a finite energy range, and is composed of a pair of branches of in- and out-of-phase subharmonic oscillations. Depending on the behavior of these VI subharmonic tongues with varying energy, the FEP is portioned into four main regimes, which are labeled as (I)-(IV) in Fig. 6.

In the highest energy regime (IV), out-of-phase VI subharmonic orbits (both symmetric and unsymmetric) bifurcate out of the backbone branch $\mathrm{S} 11-$. With increasing energy they form subharmonic tongues of out-of-phase motions with almost constant frequencies, until they reach the manifold of VI IOs (see discussion below) after which they change to in-phase motions until they coalesce with the in-phase backbone branch at specific energy 
levels; this signifies the end of these tongues and the elimination of the corresponding subharmonic motions for higher energy values. This is similar to what was observed in the FEP of the system with 'smooth' NES [57]. However, the unsymmetric subharmonic tongues do not fold on themselves to reach back to the out-ofphase backbone branch $511-$, unlike the case of smooth essential nonlinearity of the NES [57].

In regime (III) of the FEP the bifurcation behavior of subharmonic tongues is similar to regime (IV). The apparent difference is that the manifold of VI IOs undergoes a discontinuous transition on branch $S 31011-$, caused by the two bifurcations of that branch with the unsymmetric subharmonic branches $U(15) 578-$ and U8353- in that region (see Detail I in Fig. 6).

The subharmonic orbits in regime (II) exhibit different bifurcation behavior than in regimes (IV) and (III). In fact, it appears that there are no subharmonic tongues bifurcating from $S 11-$; instead, small subharmonic tongues appear to lie along the manifold of VI IOs, and eventually merge with the in-phase backbone branch $S 13+$ with decreasing energy. For example, the in-phase unsymmetric branch $U 21+$ bifurcates from $S 13+$ and transforms itself to the out-of-phase unsymmetric branch $U 21-$ after it crosses the manifold of VI IOs. In addition, for frequencies in between the two linearized frequencies $\omega_{1}$ and $\omega_{2}$ there exist multiple subharmonic branches bifurcating in a degenerate (higher co-dimensional) bifurcation from the in-phase linear mode $L 11+$ (see Detail II in the FEP of Fig. 6). These subharmonic branches coexist with the in-phase backbone branch $S 13+$, which is unstable in most of regime (II). Examples of this type of subharmonic branches are $U 2201+, U 5511+, U 4421+, \ldots$ in the FEP of Fig. 6.

Finally, the lowest energy regime (I) of the FEP is defined for energies below the bifurcation point of the linear mode $L 11-$. The manifold of VI IOs meets the stable out-of-phase linear mode L11at a bifurcation point that coincides with the critical energy level $h_{\min }=\sigma / 2$; we recall that for $h<h_{\min }$ no vibro-impacts are possible, and the dynamics of the system is completely linear.

As in the case of the system with 'smooth' NES [57], there exists a countable infinity of subharmonic tongues, corresponding to symmetric or unsymmetric VI subharmonic motions with different patterns of vibro-impacts realized during a cycle of the oscillation. Unsymmetric VI periodic orbits are represented by closed (Lissajous) curves in the configuration plane of the system. In Fig. 7(b) and(c) we depict two representative symmetric and unsymmetric VI orbits on two subharmonic branches of the FEP.

There exists a third class of VI motions in the FEP, which are denoted as VI impulsive orbits (VI IOs). These are VI periodic solutions corresponding to zero initial conditions of the system, except for the initial velocity of the LO. A VI IO represents, in essence, the response of the system being initially at rest and forced by a single impulse applied to the LO at time $\xi=0_{+}$. Apart from the clear similarity of a VI IO to the Green's function defined for the corresponding linear system, the importance of studying this class of orbits stems from their essential role regarding passive targeted energy transfer (TET) from the linear oscillator to the NES $[57,58,68,69]$. Indeed, in the case of the NES with smooth nonlinearities, IOs (which, under some conditions are in the form of nonlinear beats) play the role of bridging orbits occurring in the initial phase of TET and 'channeling' a significant portion of the applied impulsive energy from the linear primary system (in this case the $\mathrm{LO}$ ) to the NES at a relatively fast time scale; this represents the most efficient scenario for passive TET (i.e., TET through nonlinear beats $[58,68,69])$. Although the aforementioned results refer to damped impulsive orbits, the dynamics of the underlying Hamiltonian system determines, in essence, the dynamics of the damped system as well, provided that damping is sufficiently small. It follows that the IOs of the VI Hamiltonian system govern, in essence, the initial (critical) phase of TET from the LO to the NES.
The numerical results indicate that VI periodic and quasiperiodic IOs form a manifold in the FEP, containing a countable infinity of periodic IOs and an uncountable infinity of quasiperiodic IOs.

For the system under consideration, the approximation to the manifold of VI IOs was computed numerically, and is depicted in Fig. 6; in general, the manifold appears as a smooth curve, with the exception of a number of outliers (which is due to the adopted convention for the frequency index $(m: n)$ in the FEP). In each VI subharmonic tongue, a VI IO is realized whenever the relative motion between that LO and the VI NES changes from in-phase to out-of-phase. Representative VI IOs are depicted in Fig. 9. In general, the IOs become increasingly more localized to the VI NES as their energy decreases, a result which is in agreement with previous results for NESs with smooth essential nonlinearities [69]. As energy increases, the VI IOs tend towards the in-phase mode; that is, their representation in the configuration plane $\left(u_{1}, u_{2}\right)$ tends to a straight line of slope $0.084 \pi$ because the eigenvector for $L 11+$ on the $\left(u_{1}, u_{2}\right)$ plane is equal to $(1,3.702)$. Moreover, we note there is no critical energy threshold for the appearance of VI IOs since there are no low energy VI motions (the system is linear for low energy levels); the dominant frequency of a VI IO depends on the clearance $\delta$ in the physical model (i.e., on the energy regime where the VI periodic orbits exist).

Due to the degenerate VI nonlinearity of the system under consideration, it is expected that higher co-dimensional bifurcations will occur in its dynamics. One case of such degenerate bifurcations in presented in Detail II of the FEP of Fig. 6, where multiple branches of symmetric and unsymmetric VI periodic orbits (branches S95E22-, U5421-, U4520-, S55011-, U3201-, . .) are noted to bifurcate from the in-phase linear mode $L 11+$ at the point of generation of the in-phase backbone branch $S 13+$. In addition, VI periodic orbits below the branch S73E33- appear to lie along the VI IO manifold; for example, tongues U21 \pm , S95E22-, U5421-, U4520-, S55011-, U3401- and U44- in the FEP of Fig. 6.

It is interesting to note that the complexity of the FEP is solely due to the existence of the clearance $\delta$ that gives rise to vibroimpacts. Indeed, in the limit of zero clearance, $\delta \rightarrow 0$, the entire structure of VI orbits depicted in the FEP of Fig. 6 collapses to two horizontal lines corresponding to the linear modes $L 11 \pm$. We conclude that, due to the degeneracy of the VI dynamics, even a small clearance can generate significant complexity, including chaotic orbits, as discussed below.

The global dynamics of the Hamiltonian VI system (2) can be studied by constructing Poincaré maps resulting from the projection of the isoenergetic dynamics (i.e., of the dynamical flow corresponding to fixed value of $h$ ) on the two-dimensional 'cut section'

$\Sigma=\left\{\left(u_{1}, u_{2}, \dot{u}_{1}, \dot{u}_{2}\right) \in \mathbb{R}^{4} / H\left(u_{1}, u_{2}, \dot{u}_{1}, \dot{u}_{2}\right): u_{2} \equiv 0, \dot{u}_{2}>0\right\}$

which is transverse to the flow except at points where $\dot{u}_{2}=$ 0 . Similar Poincaré map construction for vibro-impact dynamics were considered in Mikhlin et al. [29]. The resulting Poincaré maps are depicted in Fig. 10. Below the energy level $h=h_{\min }=\sigma / 2=$ 0.05 no vibro-impacts occur (cf. Fig. 10(a)), and the only possible periodic solutions are the linear stable modes $L 11 \pm$.

At energy levels above this critical threshold (cf. Fig. 10(b)-(h)), vibro-impacts occur (at $\left|u_{2}-u_{1}\right|=1$ ), and generate a countable infinity of subharmonic VI orbits that replace the two linear modes $L 11 \pm$; this complex structure of orbits is a direct consequence of the non-integrability of the Hamiltonian VI dynamics. When vibroimpacts occur, the sections of the Poincare maps corresponding to $\left|u_{1}\right|>1$ are cut-off from the Poincaré maps, and the last bounding points that are included in the map are those for which the conditions $\left\{u_{2}=0\right.$ and $\left.\left|u_{1}\right|=1\right\}$ hold. For increasing energies, the 

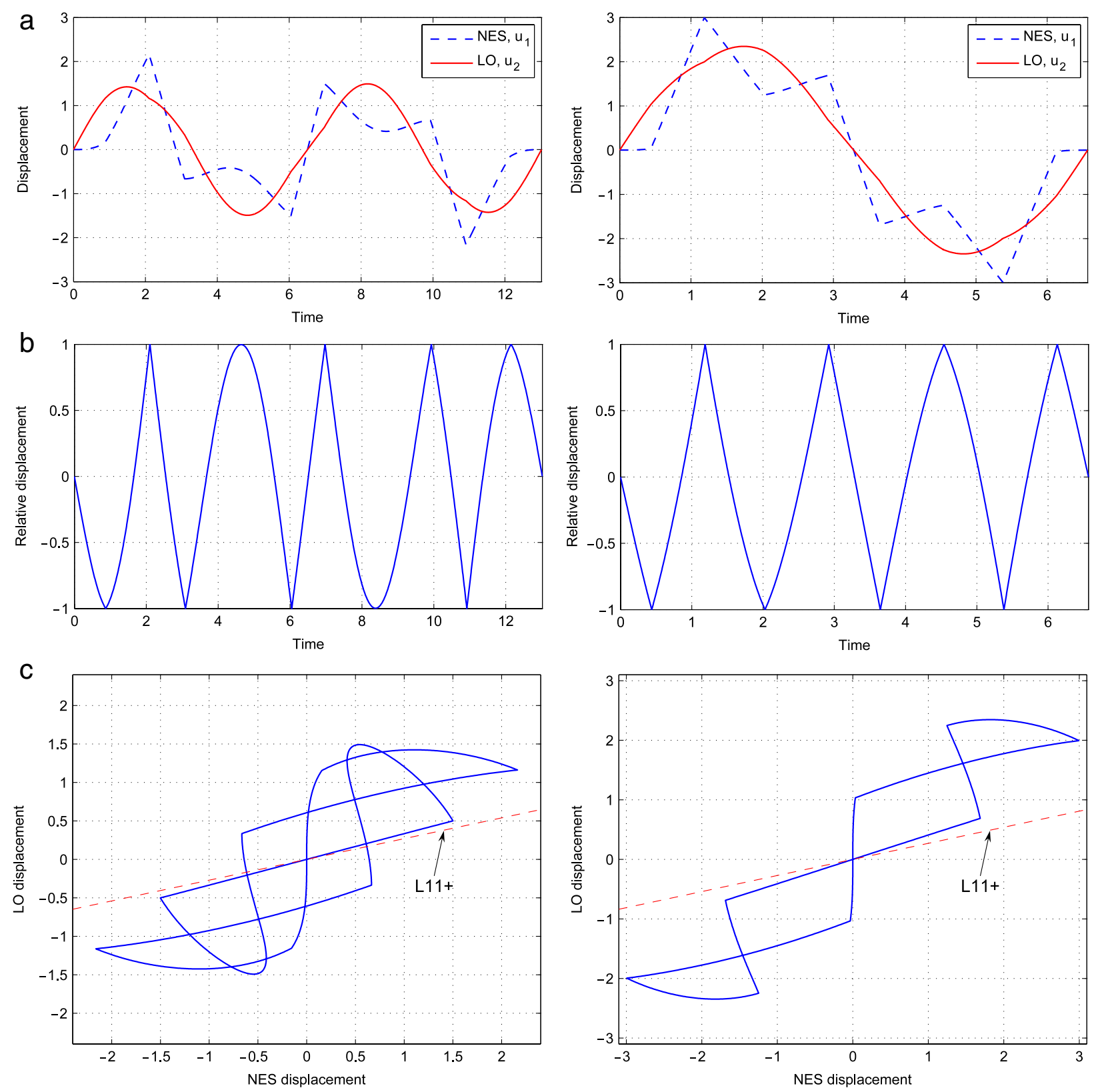

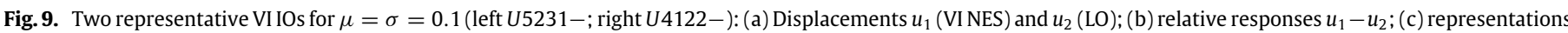
in the configuration plane $\left(u_{1}, u_{2}\right)$.

'stochastic seas' (i.e., the regions of chaotic motions) in the Poincaré maps diminish, and the domains of regular motion expand.

An additional use of the Poincaré map is that it can help us identify or infer the existence of global features of the dynamics, such as homoclinic and heteroclinic loops. For example, at the energy level $h=0.06$ (cf. Fig. 10(c)) we identify stable and unstable VI periodic orbits $U 44-$ in the neighborhood of the linear mode L11and the unstable NNM S11- (note also the location of this branch in the FEP of Fig. 6). This infers the existence of a homoclinic loop that connects the unstable periodic orbit $S 11-$. The topologies of VI IOs on branches such as U44- (which lie in the neighborhood of the SN bifurcation of S11-) are greatly influenced by the family of homoclinic orbits of the unstable branch S11- and significantly affect the efficiency of TET from the LO to the NES. This is similar to what occurs for the case of smooth nonlinearity [69], where it was found that, close to this family of homoclinic orbits, conditions for optimal TET are realized. Indeed, as shown in the next section, excitation of stable VI IOs in the neighborhood of the family of homoclinic orbits of $S 11$ - provides conditions for optimal VI TET, since large-amplitude relative displacements between the LO and the VI NES are realized in that region, and the time scale of the resulting TET is affected as well.

Apart from the compact representation of VI periodic motions, the FEP is a valuable tool for understanding the nonlinear resonant interactions (transient resonance captures or TRCs [57,58]) that govern energy exchanges and TET during damped transitions in the weakly dissipative VI system. This is due to the fact that, for sufficiently weak dissipation (caused by inelastic vibro-impacts, viscous damping or both), the damped VI dynamics is expected to be realized in neighborhoods of branches in the FEP of the underlying VI Hamiltonian system. This aspect was demonstrated in earlier work [5], where the wavelet transform (WT) spectra of the damped response of the (unnormalized) system were superimposed on the Hamiltonian FEP. The motion was initiated on a subharmonic tongue, and three distinct stages were distinguished in the resulting damped VI transition: In an initial stage, the motion 

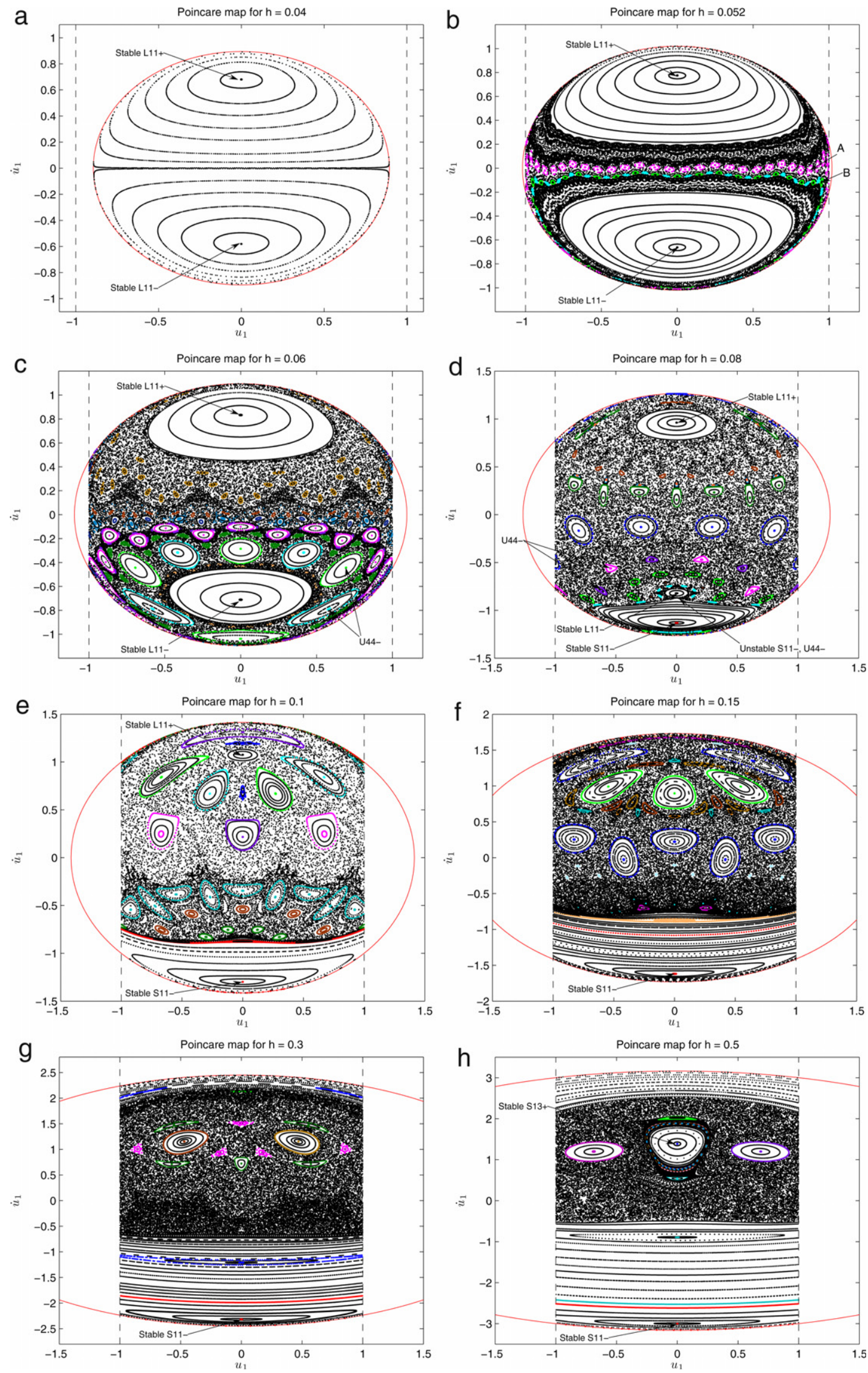

Fig. 10. Poincaré maps of VI dynamics for $\mu=\sigma=0.1$ varying energy. 
remains in the neighborhood of the subharmonic tongue where it is generated, yielding an initial persistent subharmonic TRC. As a result, subharmonic VI TET takes place from the LO to the NES, and efficient energy dissipation occurs. In the second stage of the damped motion, the dynamics makes a transition to a different lower energy subharmonic tongue, which signifies the occurrence of a different subharmonic TRC (and subharmonic TET) in the damped dynamics. Escape from this second TRC regimes leads to a transition of the dynamics to the manifold of VI IOs during the third stage of the motion, before the dynamics becomes linear, and undergoes a final transition to the linear mode $L 11+$ (the final stage of the response).

It will be shown, in the next section, that the transition of the damped dynamics along the manifold of VI IOs during the third stage of the motion is associated with a complex series of multiple TRCs with subharmonic tongues existing in the vicinity of this manifold. It follows that, by studying VI transitions in the FEP and relating them to rates of energy dissipation by the VI NES, we should be able to identify the most effective damped transitions from a TET point of view. In a more general context, we will perform a systematic study of the dynamics of TET in the two-DOF system of Fig. 3 in the next section by assuming inelastic impacts and viscous dissipation in the LO, and analyzing the resulting transient responses by numerical WTs. Then, we will superimpose the resulting WT spectra on the FEP of Fig. 6, in an effort to interpret the damped transitions in terms of the underlying Hamiltonian dynamics, and to identify the governing dynamical mechanisms for VI TET.

\section{Vibro-impact transitions in the dissipative case: VI TET}

We consider now the weakly dissipative normalized system (2) with $\lambda \neq 0$ and inelastic impacts. Then, the relations (3) that compute the normalized velocities of the LO and the VI NES immediately after an impact in terms of the corresponding velocities before impact are replaced by the following expressions

$\dot{u}_{1}^{+}=\frac{(\mu-\rho) \dot{u}_{1}^{-}+(1+\rho) \dot{u}_{2}^{-}}{1+\mu}$,

$\dot{u}_{2}^{+}=\frac{\mu(1+\rho) \dot{u}_{1}^{-}+(1-\rho \mu) \dot{u}_{2}^{-}}{1+\mu}$

where $0<\rho \leq 1$ is the coefficient of restitution. Through the numerical simulations of this section, and unless otherwise noted, we assume that $\mu=\sigma=0.1, \rho=0.7$ and $\lambda=0.005 \lambda_{c r}$, where $\lambda_{c r}=2$ is the value of critical viscous damping for the LO (hence, weak viscous damping is assumed).

Before we proceed to analyze damped transitions, we investigate the competition between the two energy dissipation sources present in the system, namely, viscous dissipation in the LO and inelastic impacts in the VI NES. For this purpose, the damped motion was initiated on the stable VI IO lying on the subharmonic tongue U8344-. In Figs. 11-13 we depict the damped responses for the following cases: (a) for no viscous dissipation in the LO and inelastic impacts ( $\rho=0.7, \lambda=0-$ Fig. 11$)$; (b) for viscous dissipation and purely elastic impacts ( $\rho=1, \lambda=0.005 \lambda_{c r}-$ Fig. 12$)$; and (c) for a combination of viscous dissipation and inelastic impacts ( $\rho=0.7, \lambda=0.005 \lambda_{c r}-$ Fig. 13). Comparing Figs. $11(\mathrm{~b}),(\mathrm{d})$ and 12(b), (d), we note distinct patterns of energy exchange and dissipation in the damped transient dynamics. For purely elastic impacts (i.e., after the last vibro-impact) the response is nearly linear and the WT spectra lie along the two linear modes $L 11 \pm$; when inelastic impacts occur, there occurs a strongly nonlinear transition of the VI dynamics along strongly nonlinear subharmonic tongues and the in-phase backbone branch $S 13+$, until, at the later stage of the response, the dynamics settles into linearized motion along the modes $L 11 \pm$. A similar, albeit weaker, nonlinear transition is noted for the case of combined inelastic impacts and viscous dissipation (cf. Fig. 13(b), (d)), where the damped dynamics traces, primarily the backbone branch (i.e., there occurs an immediate 1:1 TRC of the dynamics of the NES and the in-phase mode $L 11 \pm$ right from the beginning of the motion), and, secondarily, higher-frequency subharmonic tongues. Hence, the addition of weak viscous dissipation in the LO does not appear to affect significantly the VI damped transitions.

We focus now on the study of the mechanisms that govern VI TET by fixing $\rho=0.7$ and $\lambda=0.005 \lambda_{c r}$, in order to compare the dynamical mechanisms for VI TET to the corresponding mechanisms for the case of 'smooth' NES (i.e., with purely cubic essential stiffness nonlinearity) discussed in [58]. In the smooth case the following three mechanisms for TET were established: (a) fundamental TET, where the damped in-phase NNM invariant manifold S11+ is excited; (b) subharmonic TET, where a lowfrequency subharmonic tongue is excited; and (c) TET through nonlinear beats, where an IO close to the $1: 1$ resonance manifold of the dynamics is excited. Our study of TET in the VI case will follow similar lines, by considering energy exchanges between the LO and the NES for alternative types of initial excitation of the system. In particular, we will study VI TET when in-phase or out-of-phase periodic orbits lying on backbone and subharmonic tongues are excited, as well as when the damped motion is initiated by exciting VI IOs at various energy levels. In what follows we examine each of these cases separately.

\subsection{Fundamental VI TET}

In Fig. 14 we present the damped response of the system for initial conditions on the in-phase backbone branch $S 13+$ and initial normalized energy $h \approx 10.0$. There are four distinct stages in the damped response, which are denoted as stages A-D in Fig. 14. In the highly energetic initial stage A there occurs a 1:1 TRC in the dynamics, with the response possessing a strong harmonic at the frequency $\omega_{1}$ of the in-phase linear mode and a weaker harmonic at $3 \omega_{1}$. This is evident by examining the detailed plot depicted in Fig. 15(a), where it is clear that the relative transient response $u_{1}-u_{2}$ in stage A possesses a third harmonic component; moreover, it is noted that the WT spectrum of the relative response possesses two clear components, a main one at frequency $\omega_{1}$, and a secondary one at $3 \omega_{1}$ on the subharmonic branch $S 31011-$. This leads to fundamental VI TET from the LO to the VI NES, with almost $85 \%$ of the initial energy (nearly $40 \%$ by the LO and $45 \%$ by the VI NES) being dissipated during this initial stage of the motion. The nonlinear modal interactions that lead to fundamental VI TET will be examined in more detail later by the method of empirical mode decomposition (EMD), where the governing 1:1 TRC will be more clearly identified.

Stage B (cf. Fig. 15(b)) corresponds to a regime of pure 1:1 TRC as the third harmonic component is nearly eliminated, and the LO and the VI NES execute in-phase oscillations with frequencies approximately equal to $\omega_{1}$. It is clear that the weakly damped dynamics follows approximately the in-phase backbone branch $S 13+$ until this branch becomes unstable (i.e., at the bifurcation point where the subharmonic branches $U 11+$ and $U 21 \pm$ bifurcate out of this branch; cf. Fig. 6). This provides further evidence of the connection between the weakly damped dynamics and the dynamics of the underlying Hamiltonian system.

During stage $C$ of the damped motion there occurs a complex series of TRCs along subharmonic tongues on the FEP, whereas in the low energy stage D vibro-impacts cease to occur, the motion is purely linear, and energy dissipation is solely due to viscous damping in the LO. As expected, the linear dynamics consists of a combination of the damped analogs of the linear in-phase and 

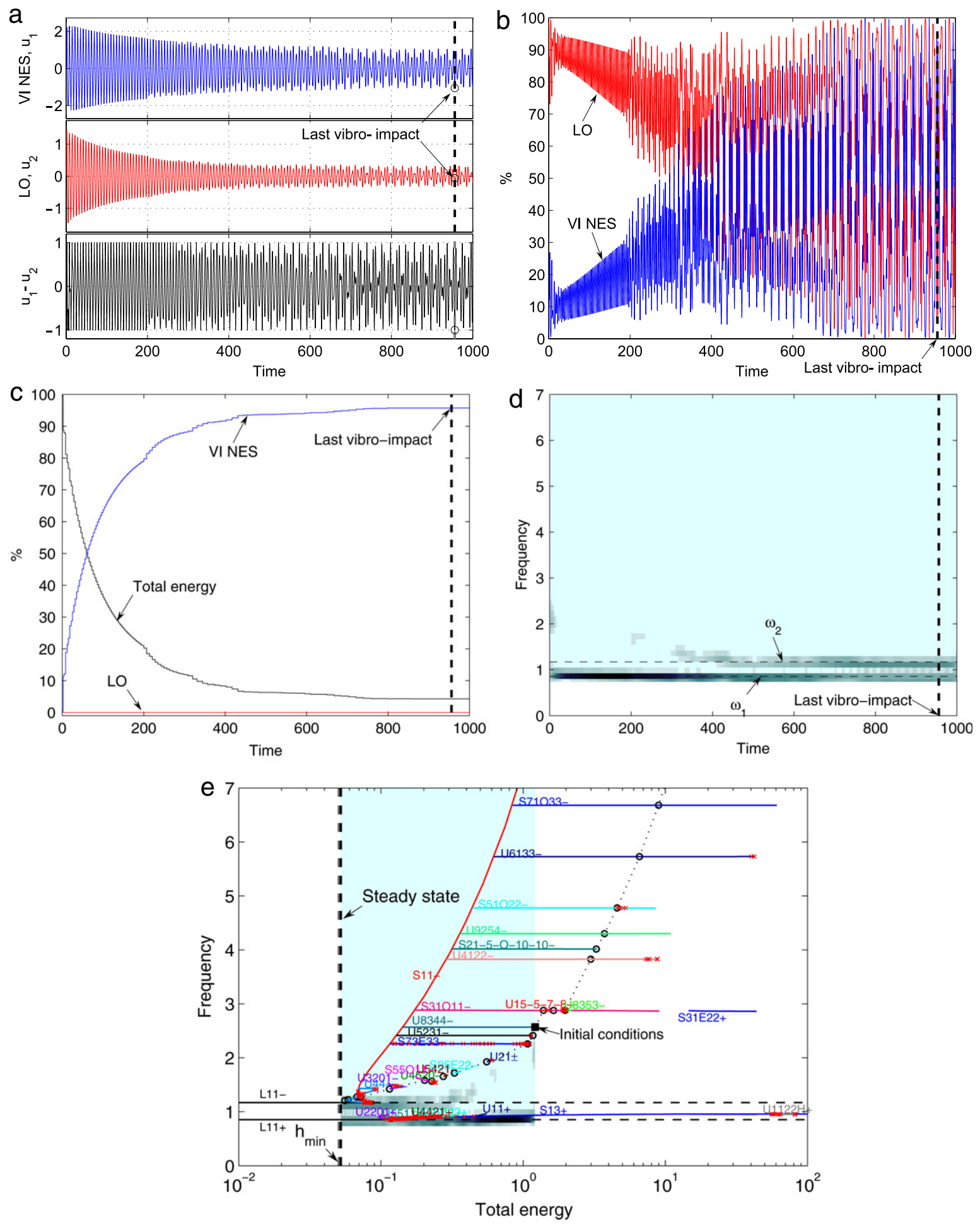

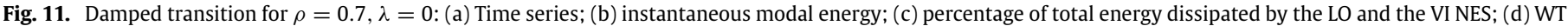
spectrum of the relative displacement $u_{1}-u_{2}$; (e) WT spectrum of $u_{1}-u_{2}$ superimposed on the FEP.

out-of-phase modes $L 11 \pm$, with mode $L 11+$ being more dominant in the response.

We conclude that in this numerical simulation there occurs fundamental VI TET due to 1:1 TRC of the dynamics of the VI NES at frequency $\omega_{1}$. Recalling that $\omega_{1}$ is the natural frequency of the inphase linear mode $L 11+$, we conclude that during fundamental VI TET the LO and the VI NES engage in in-phase 1:1 resonance capture. This VI TET mechanism is analogous to fundamental TET discussed in the case of smooth nonlinearity [58].
The next simulation examines the damped response of the system for initial conditions on the out-of-phase backbone branch $S 11-$ at $h \approx 0.8$ (cf. Fig. 16). There is insignificant TET from the LO to the NES in this case, since (as in the case of smooth nonlinearity) the initial energy of the motion localizes predominantly to the VI NES right from the beginning of the motion; then, localization to the VI NES is maintained throughout, as the damped VI motion approximately traces the backbone branch $S 11-$. In fact, in this case vibro-impacts occur only during a short initial stage of the 

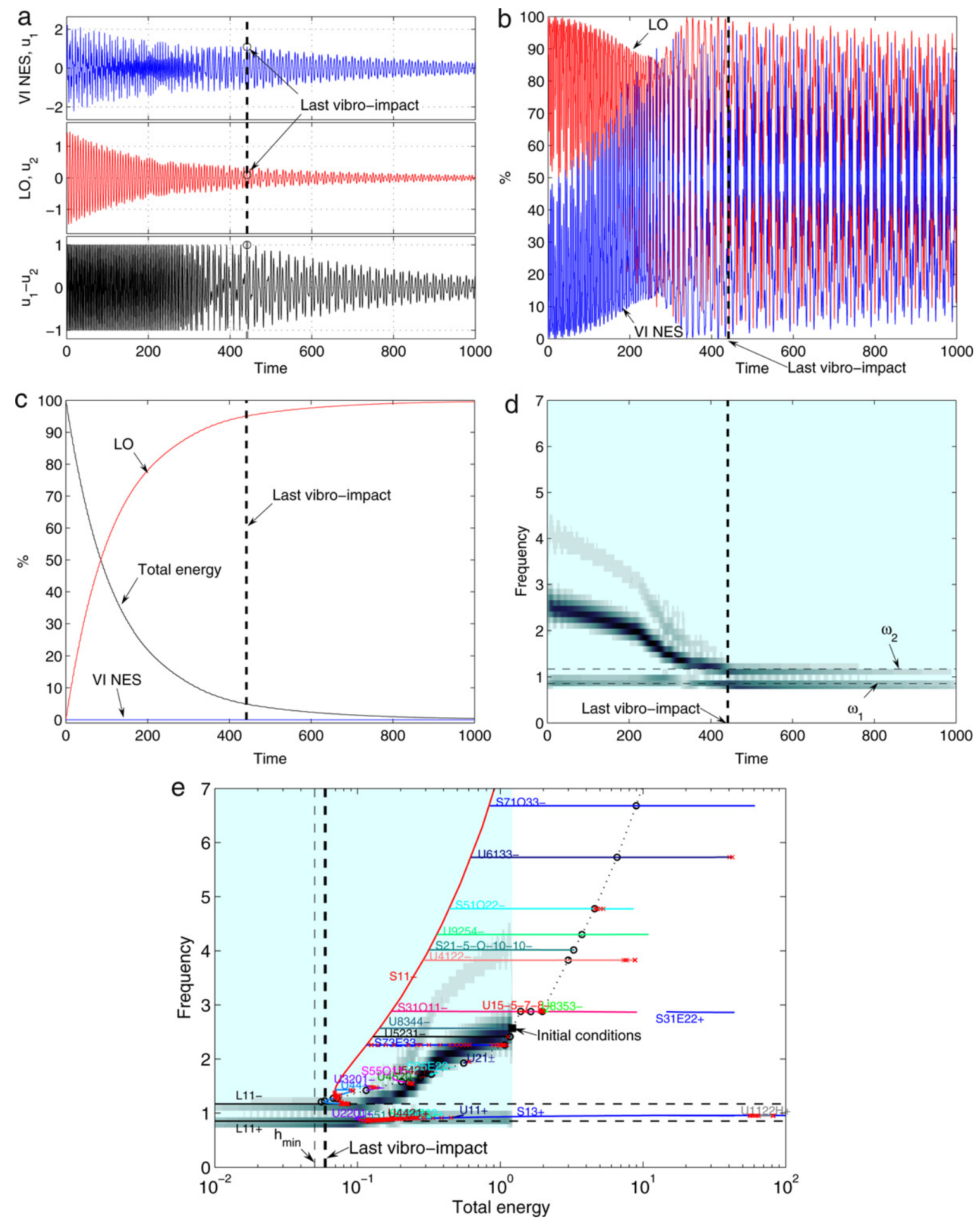

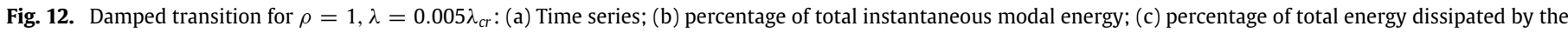
LO and the VI NES; (d) WT spectrum of the relative displacement $u_{1}-u_{2}$; (e) WT spectrum of $u_{1}-u_{2}$ superimposed on the FEP.

motion (i.e., for $\xi<10$; Fig. 16(a)), where almost $90 \%$ of total energy is dissipated. In the purely linear regime where no vibroimpacts occur (for $\xi>10$ ) the response is mainly composed of the damped analogue of the out-of-phase linear mode $L 11-$, with a weaker participation of the in-phase damped mode $L 11+$. The participation of these closely spaced modes in the linear response produces a beat phenomenon, which is evidenced by the strong energy exchanges between oscillators noted in Fig. 16(b).
We conclude that there is immediate escape of the transient damped dynamics from the initially excited out-of-phase backbone branch $S 11-$, followed by settlement of the response in alternative response regimes. This is a general conclusion drawn from the performed numerical simulations, and holds for motions that are initiated on all branches and tongues of the FEP other than the in-phase backbone branch $S 13+$ (we note that this was also the case for the case of smooth nonlinearity [58]). 

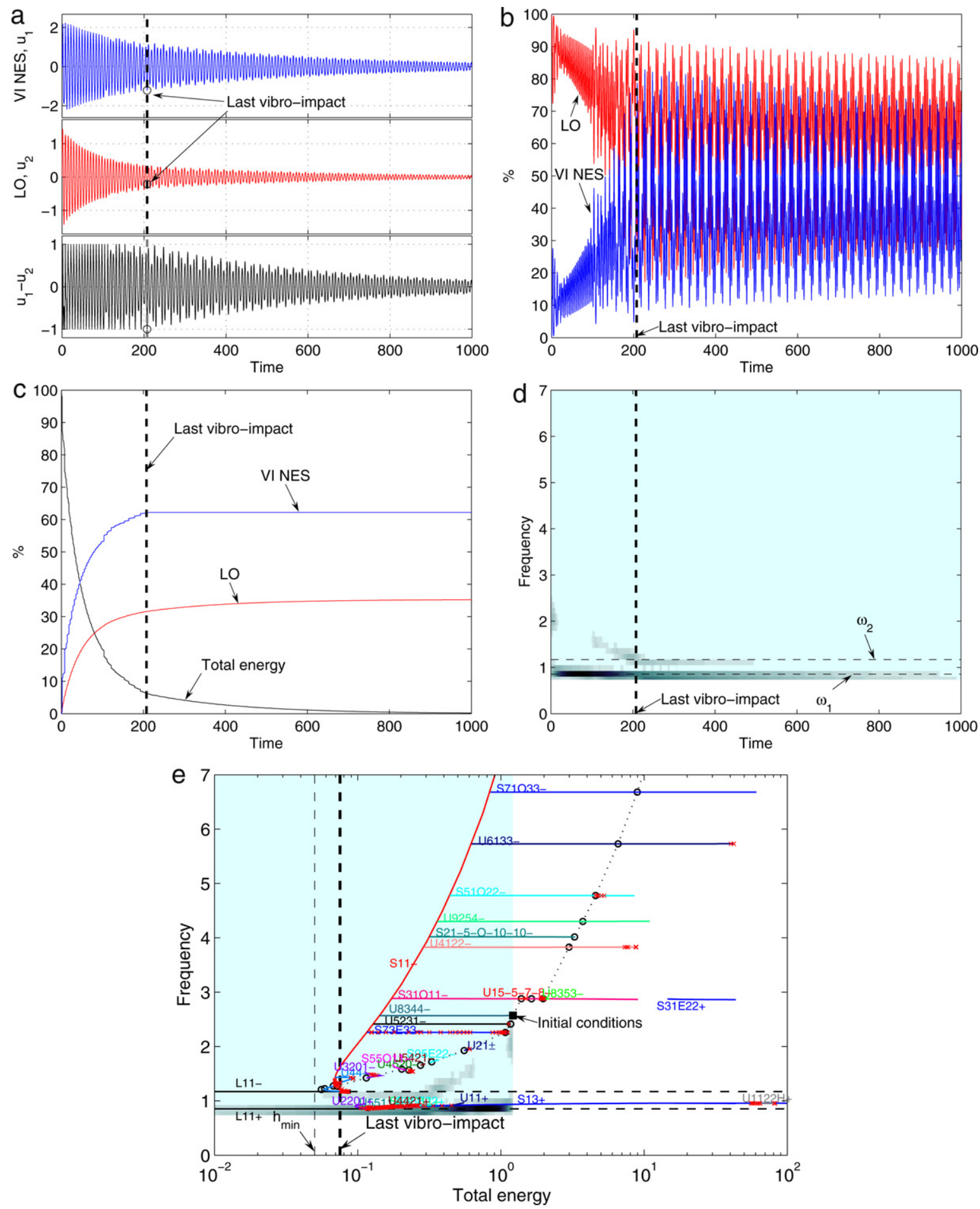

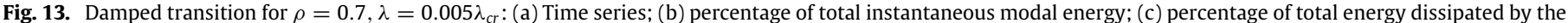
LO and the VI NES; (d) WT spectrum of the relative displacement $u_{1}-u_{2}$; (e) WT spectrum of $u_{1}-u_{2}$ superimposed on the FEP.

\subsection{TET through excitation of VI IOs}

Having established the mechanism of fundamental TET in the system with VI NES, we now consider the possibility of alternative mechanisms for VI TET based on the excitation of VI IOs. As shown in [58], excitation of IOs on certain energy ranges provides the mechanism for most efficient TET in the system with an NES with smooth nonlinearity. In the following we investigate efficiency of TET from the LO to the NES when VI IOs are excited in the four previously defined regimes (I)-(IV) of the FEP. We note that in the case of NES with smooth nonlinearity IOs play an important role as far as TET is concerned; this holds especially for IOs lying in the neighborhood of the family of homoclinic orbits of the unstable out-of-phase damped NNMs $S 11-$, close to the 1:1 resonance manifold of the damped dynamics [69].

In Fig. 17 we depict the damped response of the system when a VI IO in regime (I) is excited. Since the initial energy of the motion is relatively low, vibro-impacts occur only during the shortduration initial stage of the dynamics, and afterwards the dynamics become completely linear, involving continuous energy exchanges 

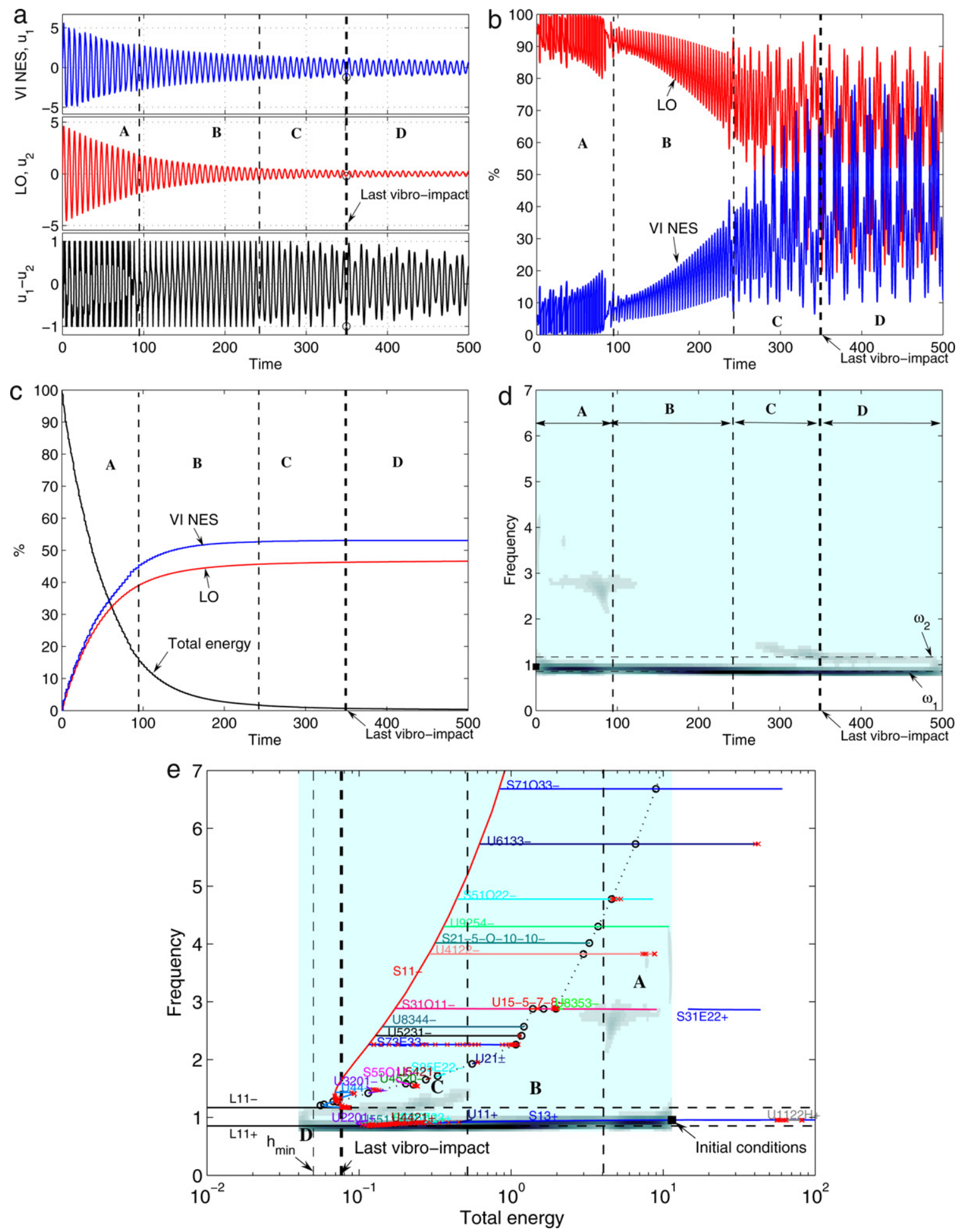

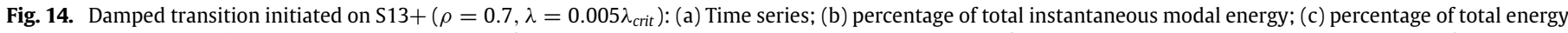

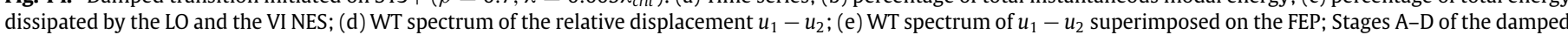
transition are indicated.

between the two linear modes of the system (with no vibroimpacts) at frequencies $\omega_{1}$ and $\omega_{2}$. Due to the closely spaced linear natural frequencies, a linear beat develops and energy is predominantly dissipated by viscous dissipation in the LO. In this case insignificant TET from the LO to the VI NES occurs.

The damped responses for initial excitation of a VI IO on the subharmonic tongue S95E22 - in regime (II) of the FEP are depicted in Fig. 18. In this case the dynamics cannot exhibit a 1:1 TRC, since the in-phase backbone branch $S 13+$ is unstable at the specific initial energy level considered in this simulation. As a result, the damped dynamics may be divided into four distinct stages, labeled by A-D in Fig. 18. Stages A-C are strongly nonlinear, whereas, the low energy stage $D$ is linear with no vibro-impacts occurring there. In stage $A$ the damped dynamics follows approximately the tongue S95E22 - (where the motion is initiated) with decreasing energy. Nearly $50 \%$ of the total initial energy is dissipated during this Stage 

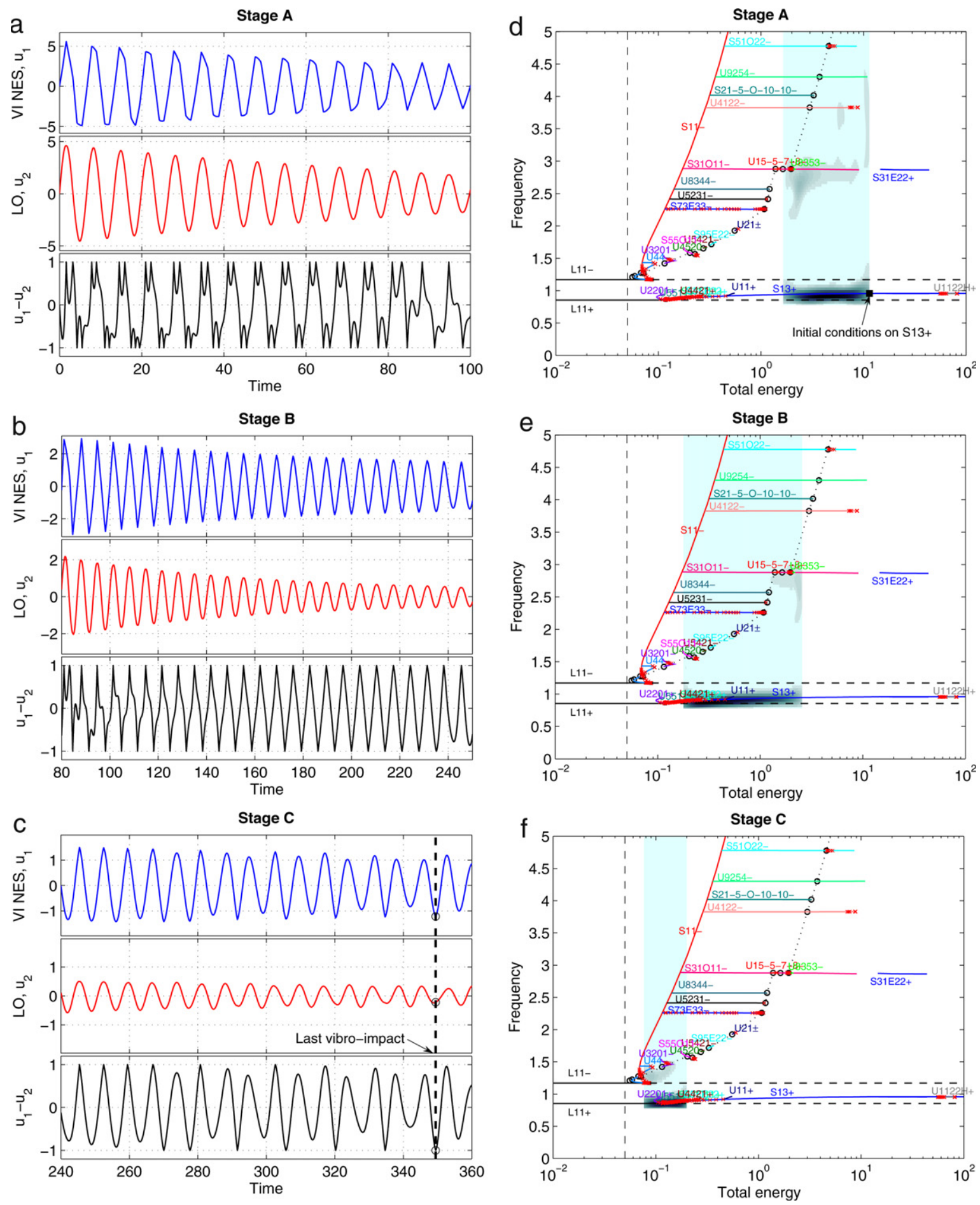

Fig. 15. Close-ups of the time series (left column) and WTs on the FEP (right column) depicted in Fig. 14.

of the response, with $33 \%$ of total energy being dissipated due to TET from the LO to the VI NES. The damped dynamics in stages B and $\mathrm{C}$ is complex, as it undergoes transitions along subharmonic tongues such as U5421-, U4520-, S55011- and U3201- lying close to the manifold of IO. Finally, when sufficient energy is dissipated and no additional vibro-impacts can occur the dynamics settles into the linear stage $D$, where predominant contribution of mode $L 11+$ is realized.

In Fig. 19 the damped dynamics for excitation of the VI IO on the subharmonic tongue $S 31011$ - in regime (III) of the FEP is presented. For the selected initial energy level for this simulation, $1: 1$ TRC is possible (since the backbone branch $S 13+$ is stable at the initial energy level considered), and five distinct stages of the damped motion (labeled as A-E in Fig. 19) are inferred. In stage A the damped motion follows the subharmonic tongue S31011which acts as bridging orbit for the dynamics to make the transition from its initial state to $1: 1$ TRC (which is realized in stage $B$ ). In stage $B$ there occurs a 1:1 TRC as the stable in-phase backbone branch $S 13+$ is excited; as a result, fundamental TET from the LO to the VI NES is realized, so that nearly $50 \%$ of the total initial energy is dissipated by the VI NES by the end of this stage of the motion. As energy decreases due to viscous dissipation and inelastic impacts, the in-phase backbone branch $S 13+$ becomes unstable and the damped dynamics makes a transition to stages 

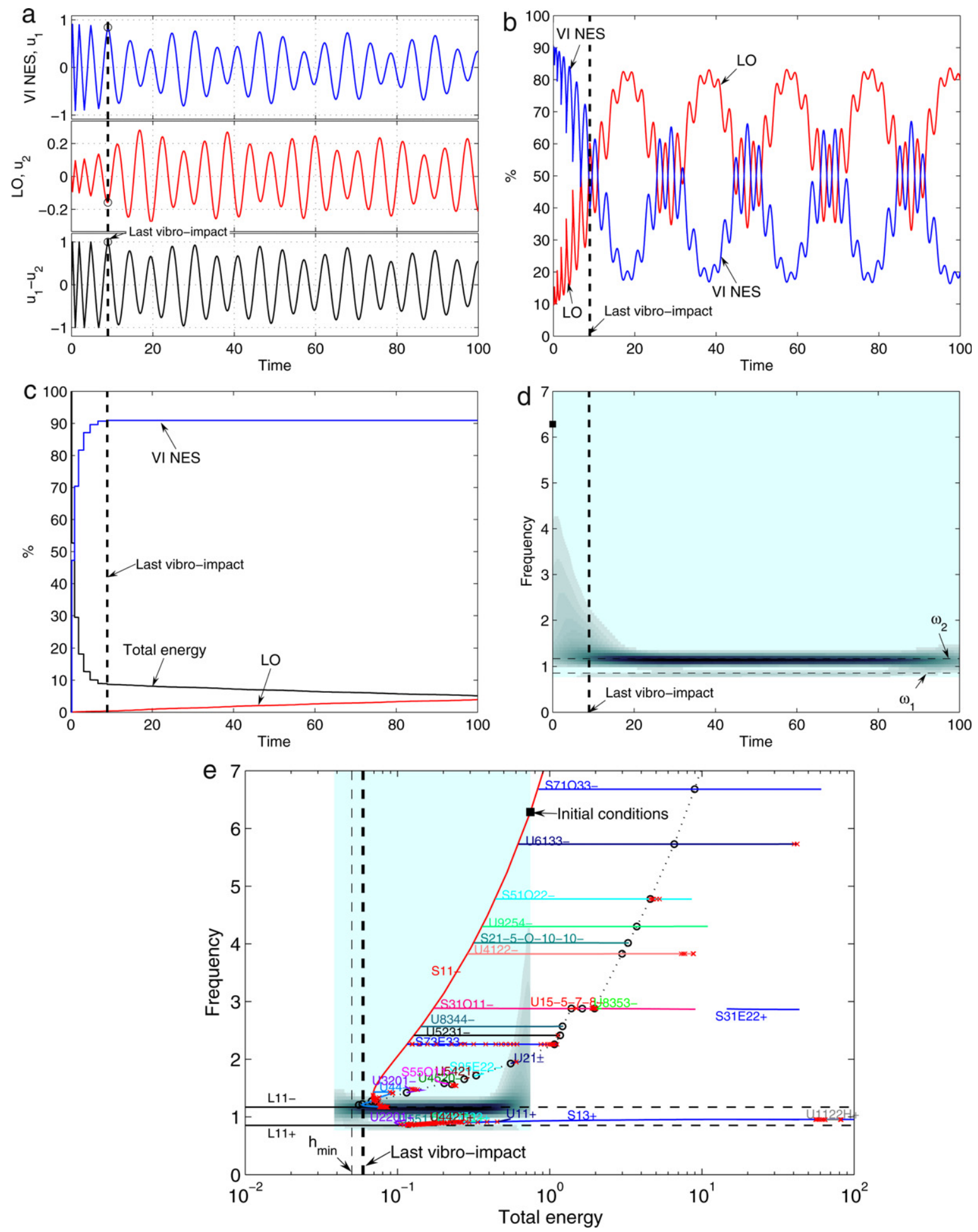

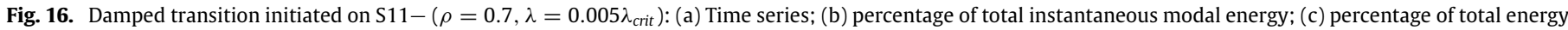
dissipated by the LO and the VI NES; (d) WT spectrum of the relative displacement $u_{1}-u_{2}$; (e) WT spectrum of $u_{1}-u_{2}$ superimposed on the FEP.

$\mathrm{C}$ and $\mathrm{D}$; these stages are similar to those occurring in regime (II) of the FEP, and the dynamics follows complex transitions along subharmonic tongues, similar to the ones depicted in Fig. 18. At the later, low energy stage $E$ the dynamics is linear and dominated by mode $L 11+$. We conclude that by exciting VI IOs lying in Regime III of the FEP the 'bridging orbit' scenario is realized (as in the case of the system with 'smooth' NES), leading eventually to the fundamental VI TET. This scenario yields efficient TET from the LO to the VI NES.
Finally, when IOs in the high energy regime (IV) of the FEP are excited (cf. Fig. 20) the damped transitions are similar to those realized in regime (III), with TET efficiency at the end of fundamental VI TET reaching the level of nearly $55 \%$.

In conclusion, we identify two mechanisms for VI TET, namely, fundamental VI TET due to 1:1 TRC and VI TET through excitation of a VI IO leading eventually to fundamental VI TET. These are similar to the corresponding TET mechanisms for the case of 'smooth' NES. No subharmonic VI TET (caused by TRC of the dynamics on an 

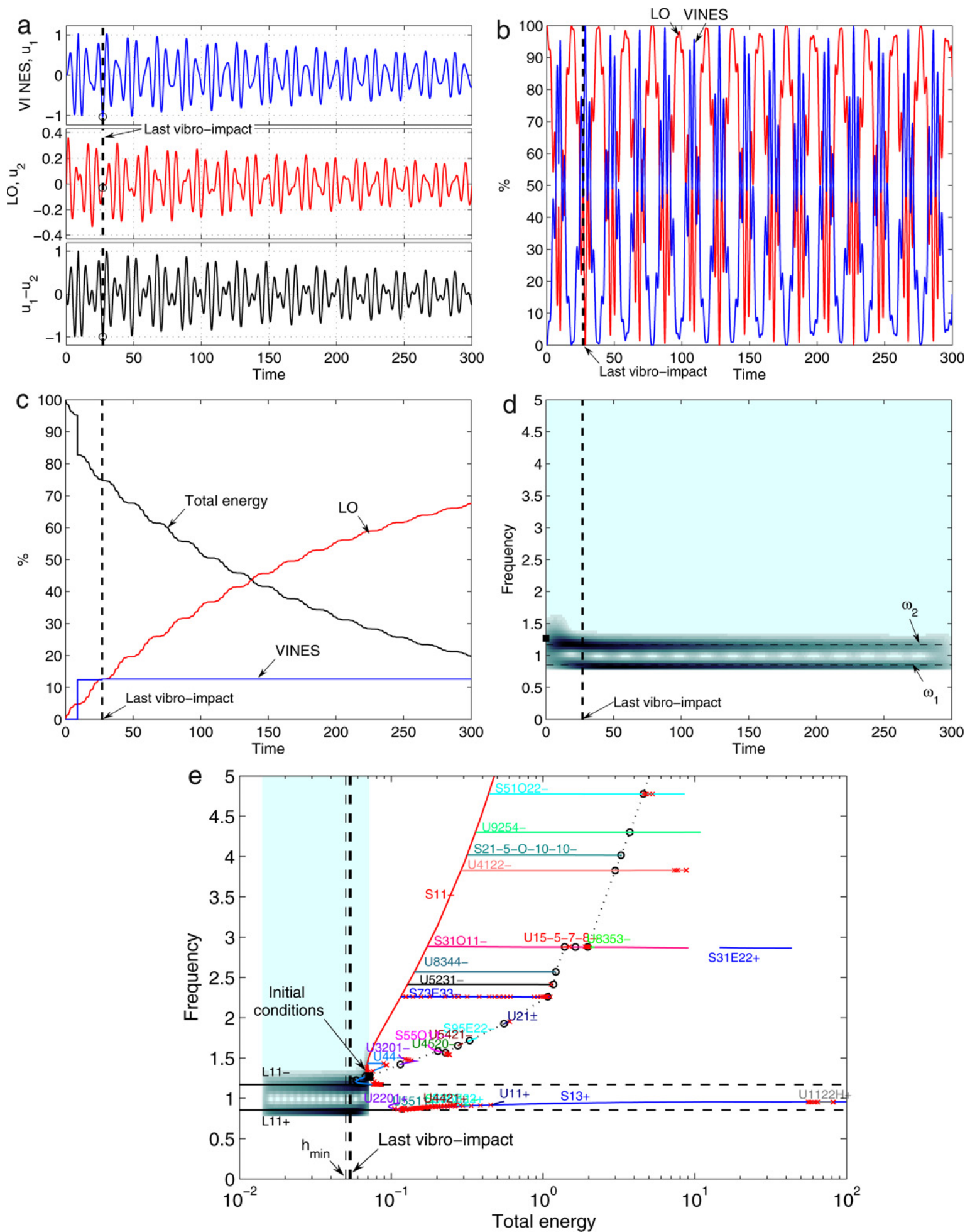

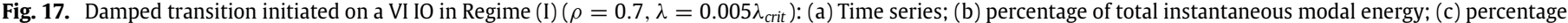
of total energy dissipated by the LO and the VI NES; (d) WT spectrum of the relative displacement $u_{1}-u_{2}$; (e) WT spectrum of $u_{1}-u_{2}$ superimposed on the FEP.

isolated VI subharmonic tongue) could be realized in the numerical simulations of the dynamics of the VI system under consideration, as the VI dynamics seem to engage in series of TRCs involving multiple subharmonic tongues (instead of an isolated one) lying close to the manifold of VI IOs. However, as shown in the simulations of [5], subharmonic VI TET is indeed possible in the VI system of Fig. 3. Therefore, we conjecture that subharmonic VI TET can be a mechanism for TET in systems with very weak viscous damping and weakly inelastic impacts. A final conclusion drawn from the previous simulations is that lack of fundamental TET in regimes (I) and (II) of the FEP can be attributed to the instability of the in-phase backbone branch $\mathrm{S} 13+$ in the corresponding energy ranges. This is an additional indication of the strong relation that exists between the Hamiltonian dynamics and the weakly damped transitions. 

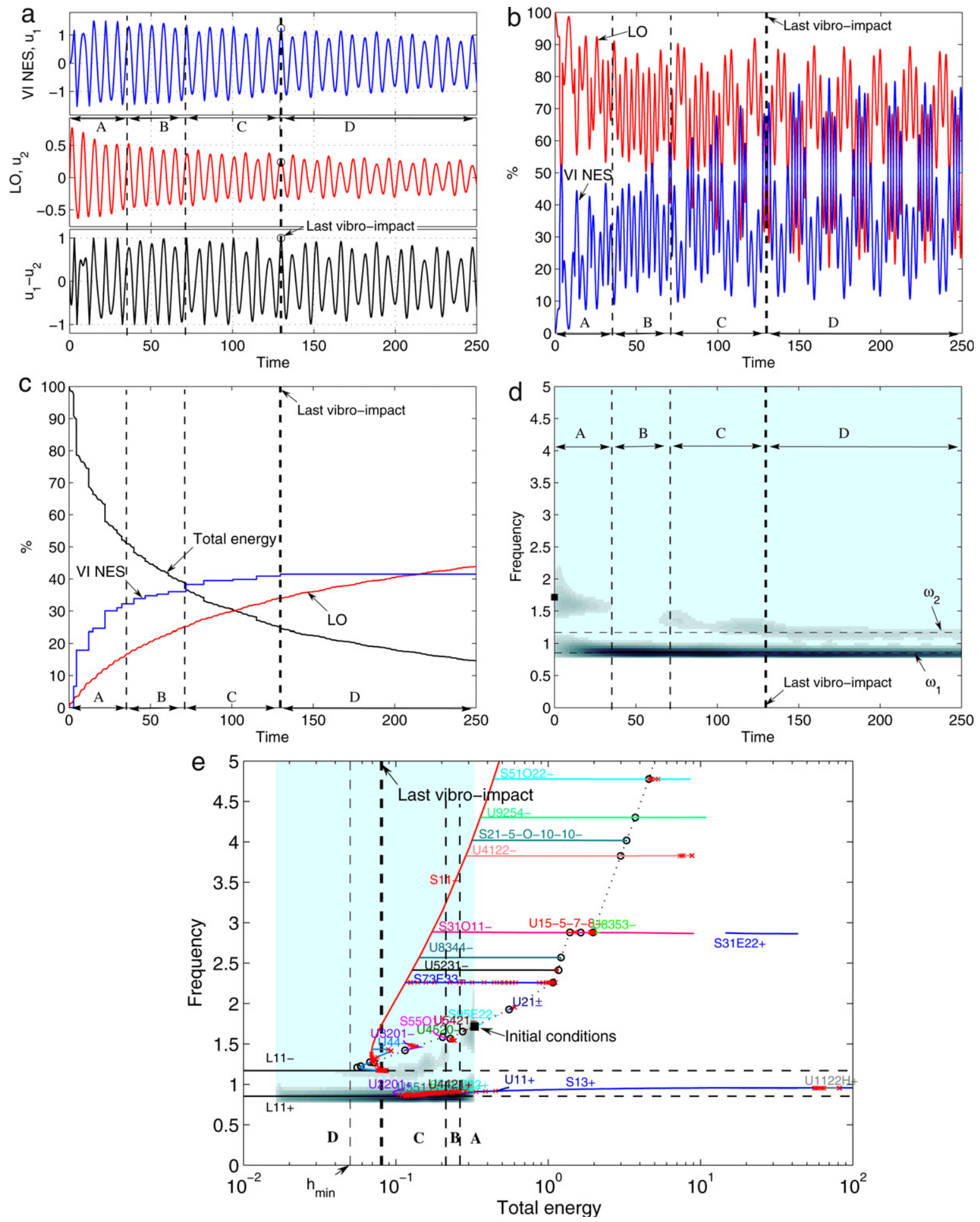

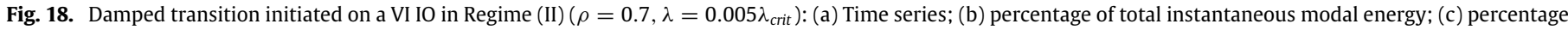
of total energy dissipated by the LO and the VI NES; (d) WT spectrum of the relative displacement $u_{1}-u_{2}$; (e) WT spectrum of $u_{1}-u_{2}$ superimposed on the FEP.

\subsection{Fundamental VI TET mechanism by means of empirical mode decomposition (EMD)}

The specific nonlinear resonance interactions that lead to TET in the VI system can be analyzed through EMD (for example, see [70]). We demonstrate this by analyzing in detail the mechanism for fundamental VI TET and showing that it is a 1:1 TRC. To this end, we analyze the high energy damped transition of Fig. 14 for the motion initiated on the in-phase backbone branch $S 13+$.
Decomposition of nonlinear damped transitions by EMD leads to multi-scale nonlinear identification of the governing dynamics, and provides the means for interpreting nonlinear resonance (modal) interactions between coupled oscillators, as well as the time (or frequency) scales where these modal interactions occur [71].

In Fig. 21 we depict the results of EMD analysis utilizing Matlabbased codes [72], from which we conclude that both responses of the LO and VI can be decomposed into two intrinsic mode functions (IMFs) (Fig. 21(a) and (b)), respectively. Thus, the exact 

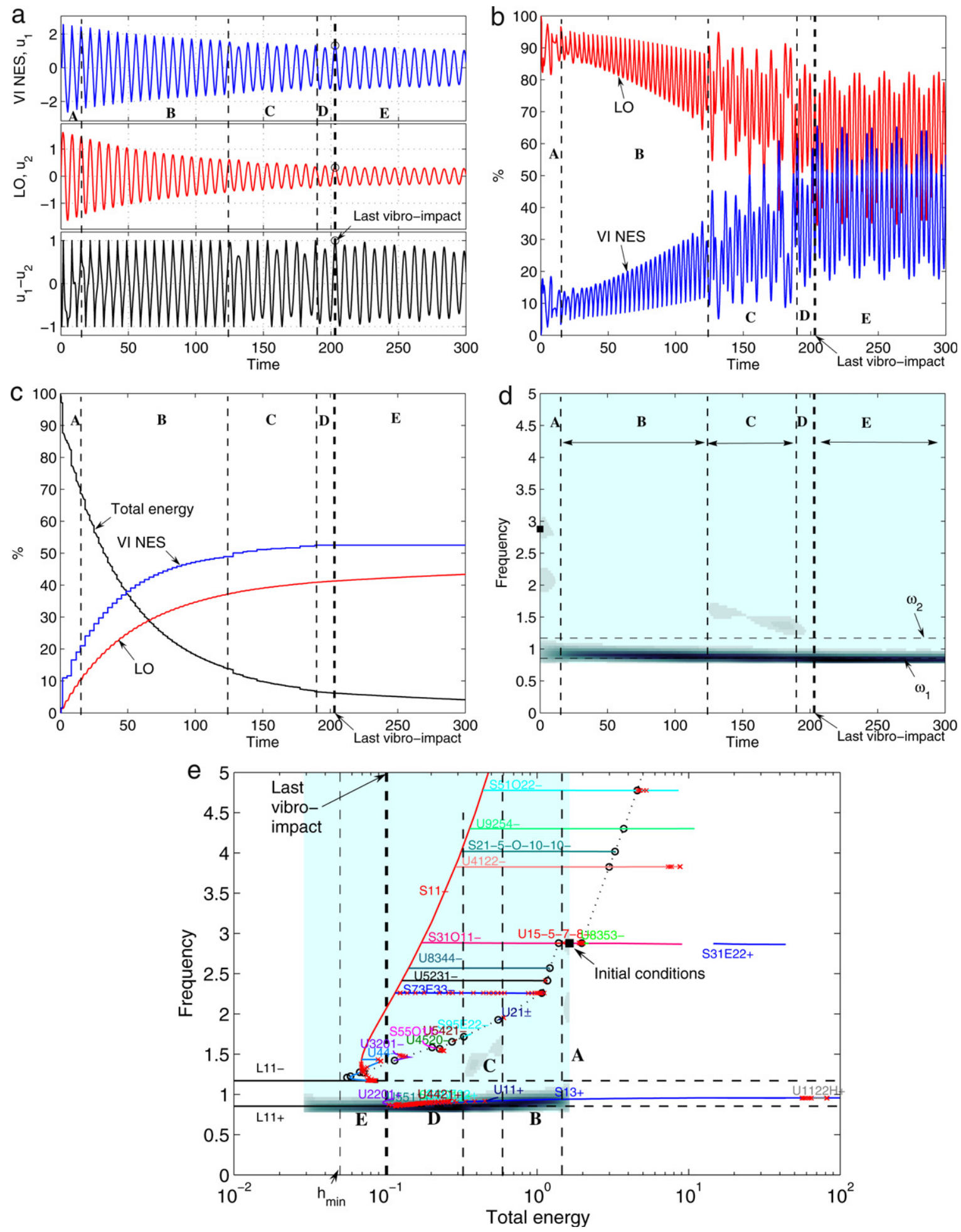

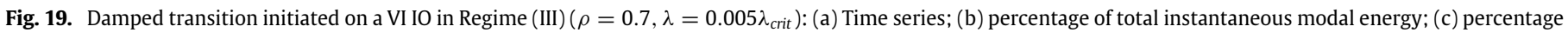
of total energy dissipated by the LO and the VI NES; (d) WT spectrum of the relative displacement $u_{1}-u_{2}$; (e) WT spectrum of $u_{1}-u_{2}$ superimposed on the FEP.

responses can be accurately reconstructed with the second IMFs, which capture the dominant frequency components of the original time series (Fig. 21(c)). It is interesting to observe that the first IMFs for both oscillators detect all occurring impacts, so that the second IMFs are smooth functions possessing frequency components completely free of nonsmooth effects due to vibro-impacts. Fig. 22 provides a more detailed study of the first IMFs of the LO and the VI NES, from where the patterns of vibro-impacts at different stages of the damped response can be clearly inferred; indeed, one can detect that the motions of both oscillators are out-ofphase whenever vibro-impacts occur. This observation explains (i) the enhanced energy dissipation due to inelastic vibro-impacts in the system considered, and (ii) the reason behind the fact that vibro-impacts hardly contribute to the observed nonlinear resonance interactions, as these occur in-phase and involve mainly $1: 1$ resonance captures. This suggests that the seemingly $3: 1$ resonant interaction (particularly at the initial TET stage) indicated by the WT spectra superimposed to the FEP (Fig. 14(e)) may be, in 

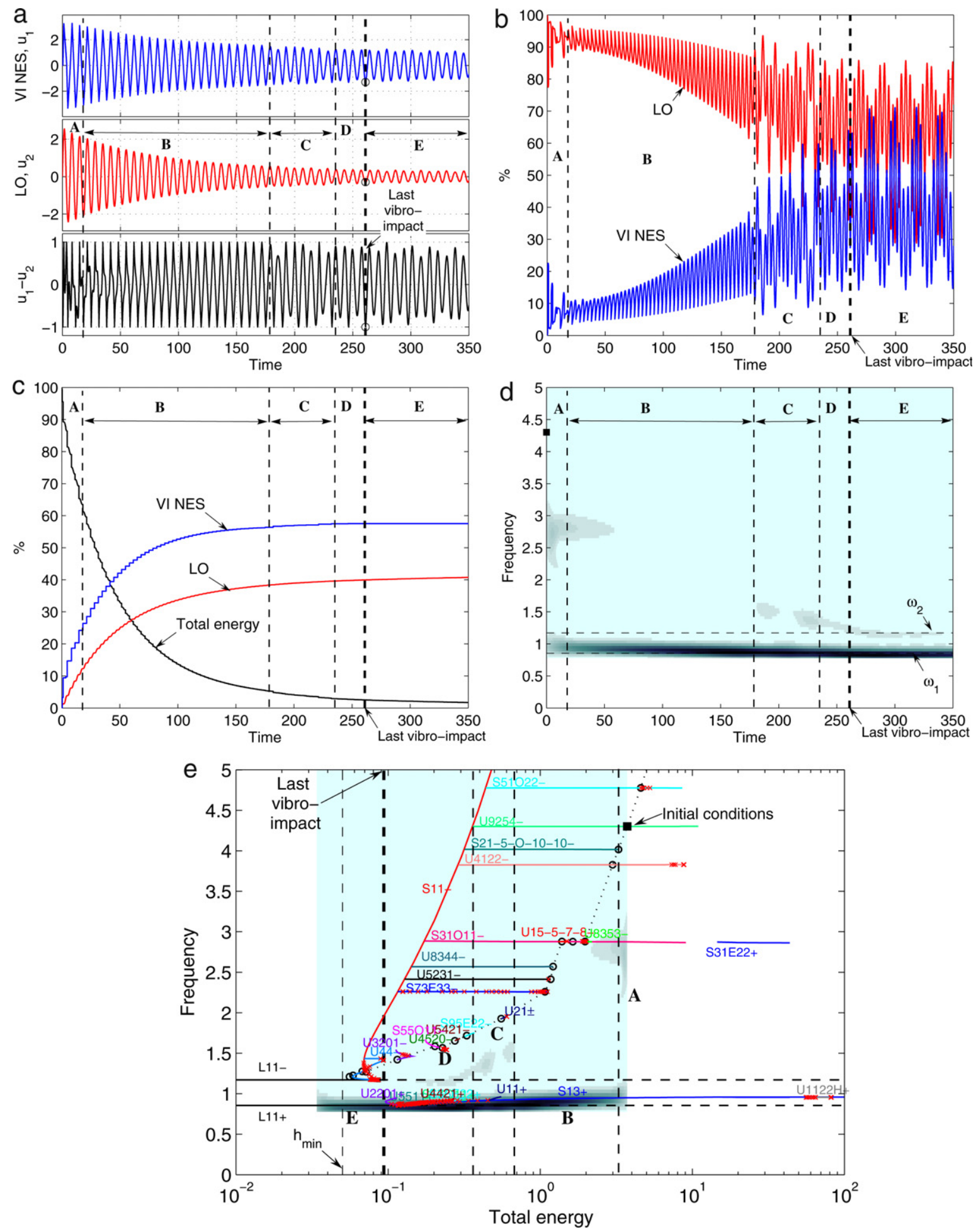

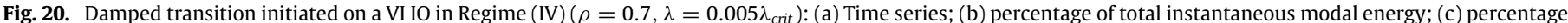
of total energy dissipated by the LO and the VI NES; (d) WT spectrum of the relative displacement $u_{1}-u_{2}$; (e) WT spectrum of $u_{1}-u_{2}$ superimposed on the FEP.

fact, purely 1:1 TRC following the in-phase NNM branch $S 13+$ from the start.

Before demonstrating this latest argument by Hilbert spectral analysis, we compute the (averaged) frequency of vibro-impact occurrences in Fig. 23. First, the period of vibro-impacts on average can be estimated by $T_{i}=\xi_{i+1}-\xi_{i}, i=1,2, \ldots, N-1$ where $N$ is the total number of impacts. Then, its inverse $1 / T_{i}$ can be regarded as the averaged impact frequency; and the corresponding circular frequency can be computed by multiplying this result by
$2 \pi$. The period and frequency of vibro-impacts evaluated this way are assumed to correspond to the instants at $\left(\xi_{i+1}-\xi_{i}\right) / 2, \forall i$. From Fig. 23, we observe that the three distinct VI TET regimes are clearly represented by these two quantities. In stage A which dictates the initial VI TET period, fluctuations of impact frequency in the high-frequency regime above 3 are observed; that is, occurrence of vibro-impacts is irregular. On the other hand, vibro-impacts occur almost at a constant rate during stage $\mathrm{B}$ (at circular frequencies around 1.5); and in stage $C$ the vibro-impacts exhibit a more 

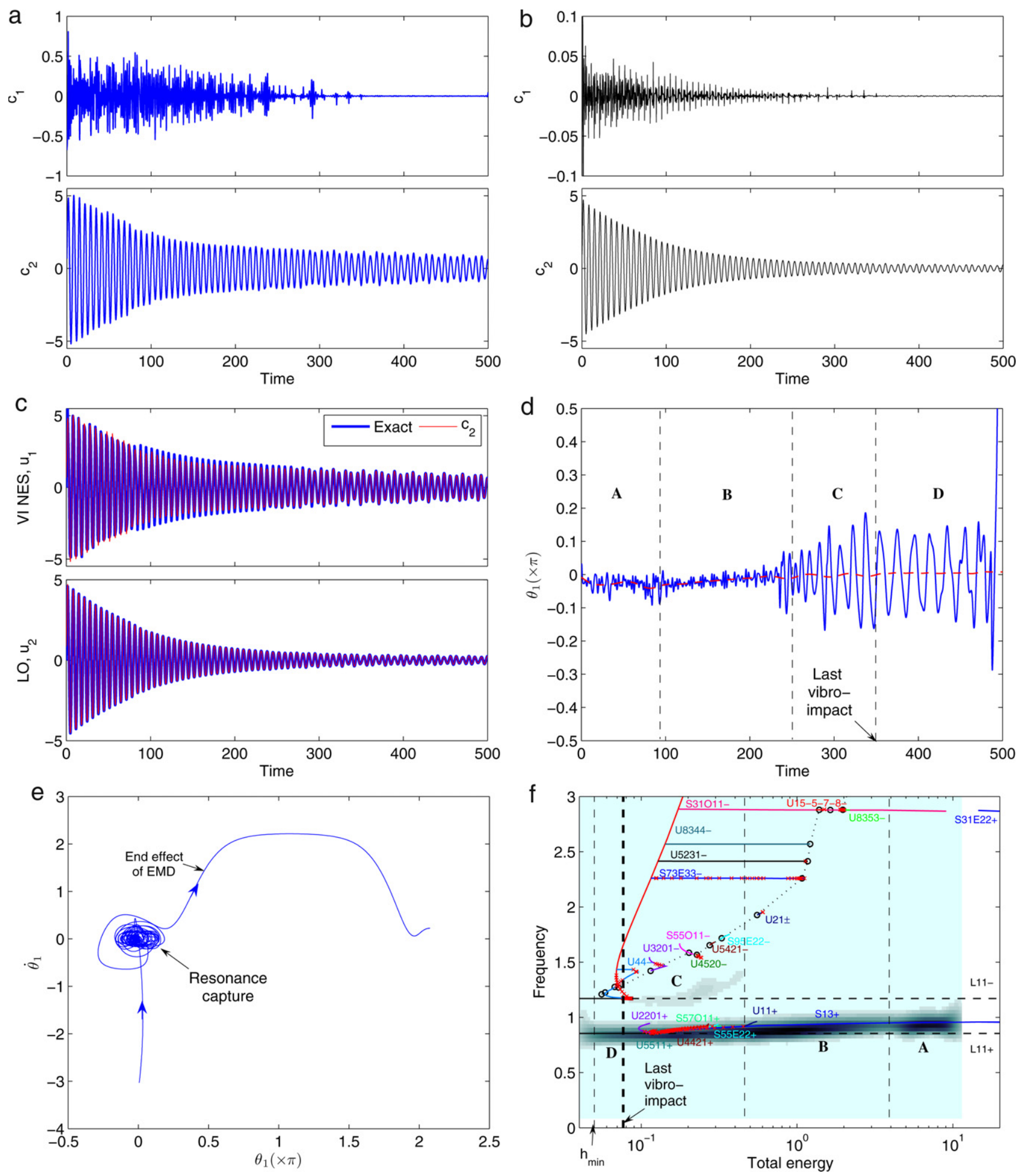

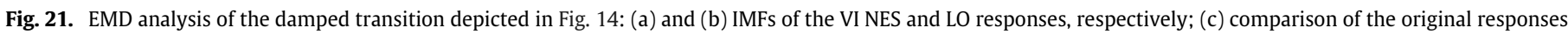

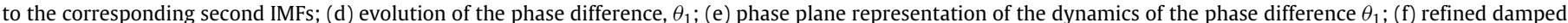
transition of the FEP where only the second IMFs of the responses are considered.

irregular pattern. In particular, the irregular occurrence of vibroimpacts at the initial stage of the damped transition can be another evidence that the 3:1 resonant interaction at stage $A$ is, in fact, a numerical artifact exclusively due to impacts, whereas in actuality the LO and the VI NES are engaged in 1:1 in-phase transient resonance capture.

Recalling that only the second IMFs represent accurately the exact responses of the LO and the VI NES, we denote by $\phi_{1}$ the instantaneous phase of the second IMF of the VI NES response computed by application of the numerical Hilbert transform; similarly, we denote by $\phi_{2}$ the corresponding phase of the second IMF of the LO response; finally, we define $\theta_{1}=\phi_{1}-\phi_{2}$ as the phase difference between the two oscillators. Then, TRCs occurring between the second (dominant) IMFs of the responses of the two oscillators can be studied in detail. Indeed, when a specific phase difference exhibits time-like (i.e., monotonic with time) behavior 

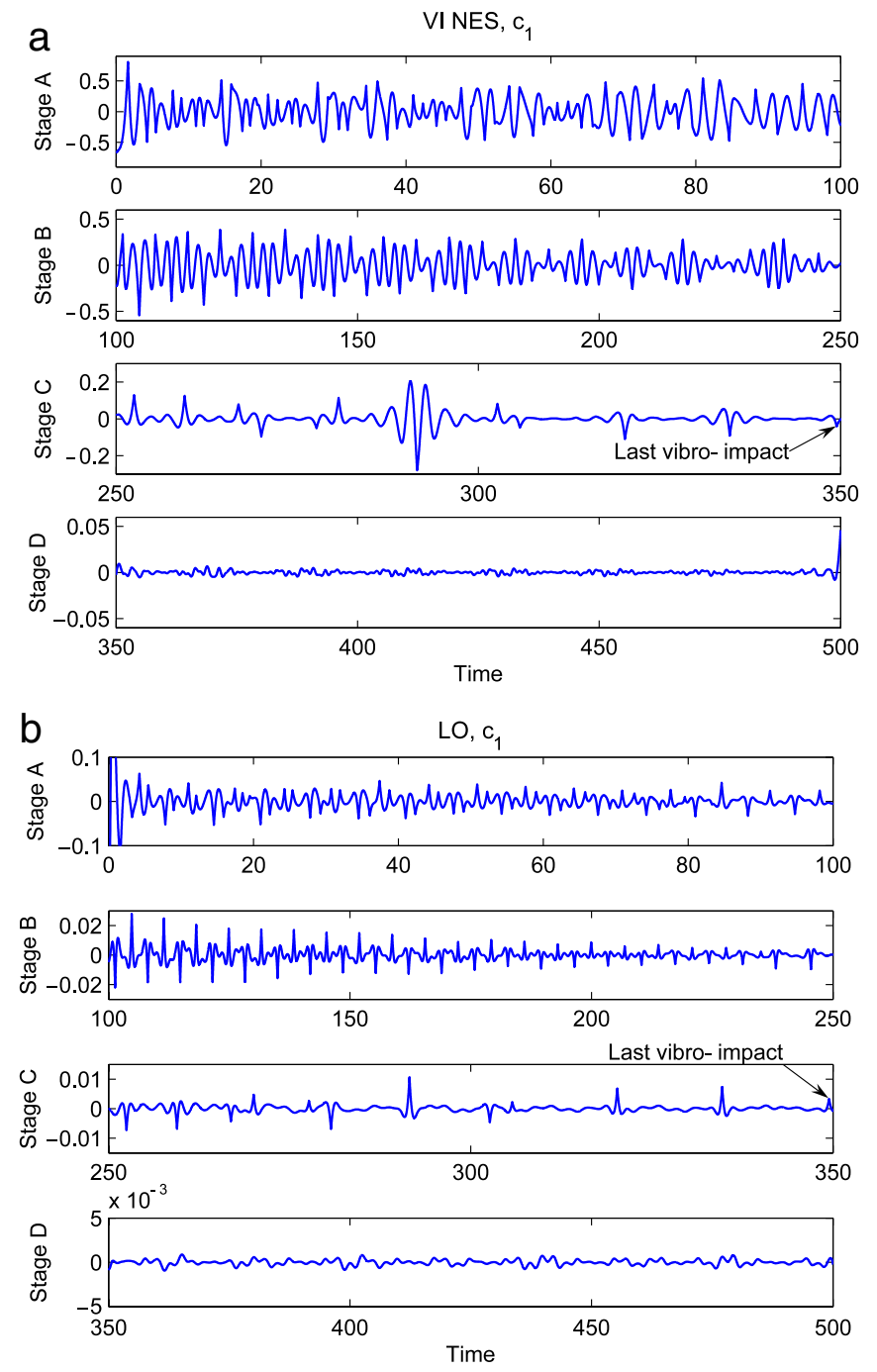

Fig. 22. Detailed depiction of vibro-impacts in the first IMFs of Fig. 21.
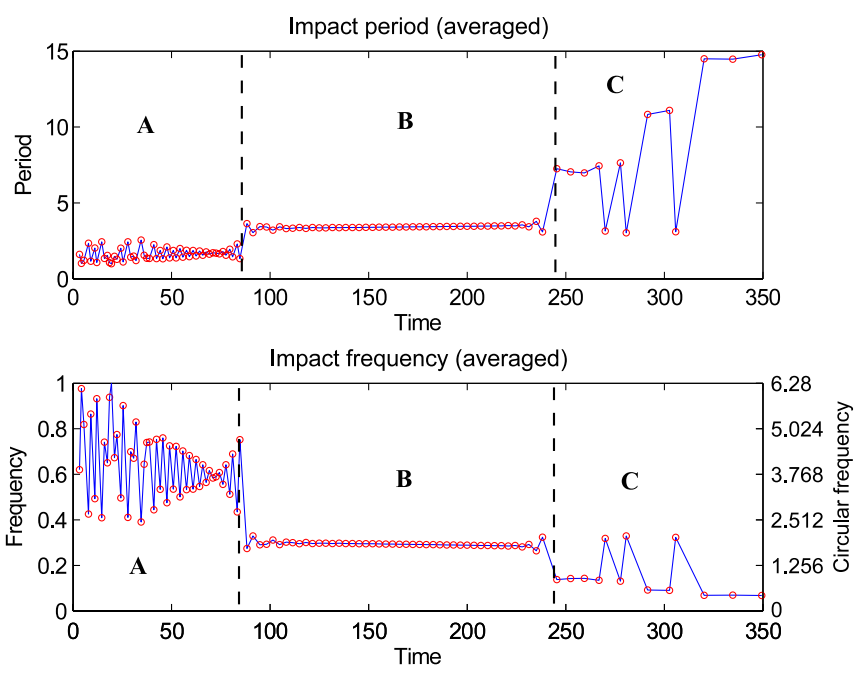

Fig. 23. Period and frequency of impacts.

over a specified time interval, it may be regarded as a 'fast' angle, and, hence, may be averaged out of the dynamics; this eliminates the possibility of resonance interaction between the corresponding IMFs. On the contrary, non-time-like behavior of a phase difference precludes the direct application of the averaging theorem with respect to that angle, and the possibility for resonance interaction between the corresponding IMFs exists.

In Fig. 21(d) we note non-time-like behavior of the phase difference during the entire VI damped motion, which indicates that a 1:1 TRC between the two oscillators occurs. The phase plane $\left(\theta_{1}, \dot{\theta}_{1}\right)$ in Fig. $21(\mathrm{e})$ more clearly depicts this non-time-like behavior, indicated by the spirals in the dynamics during the entire regime of the VI transition. Finally, the wavelet transform of the difference between the two second IMFs of the two oscillators, superimposed on the FEP, clarifies the true nonlinear resonant interactions without being 'polluted' by the occurring vibro-impacts (Fig. 21(f)). It is interesting to note that the EMD results can separate effects of vibro-impacts from the occurring nonlinear resonance interactions, so that the residuals represent smooth functions that describe true nonlinear resonant interactions between the two oscillators. This demonstrates that the EMD technique, although applied (by construction) in an ad hoc manner, can still lead to physically relevant results. Currently, efforts towards a rigorous physical interpretation of EMD results in terms of the slow-flow dynamics of a system, and application of EMD in the context of nonlinear nonparametric system identification are in progress [71].

We end this section by mentioning that the presented VI TET results are by no means optimized; that is, higher TET efficiencies may be achieved when alternative sets of initial conditions or system parameters are considered. This leads us naturally to the discussion of TET efficiency in the system with VI NES carried out in the next section.

\section{Efficiency of VI TET}

We aim to study the efficiency of VI TET in the system of Fig. 3 by introducing certain definitions related to the capacity of the VI NES to passively absorb and dissipate vibration energy from the LO, as well as the time required for this VI dissipation to occur. Specifically, we denote by $\xi_{L I}$ the normalized time instant when the last vibro-impact in a given simulation occurs (that is, for $\xi>\xi_{L I}$ the transient response is purely linear); by $\xi_{95 \%}$ the time required for $95 \%$ of the initial energy of the system to get dissipated by viscous damping and inelastic vibro-impacts; and by $E_{V I N E S}$ the percentage of initial energy eventually dissipated by the VI NES due to inelastic vibro-impacts (i.e., during the entire duration of the damped motion). In this context, the ratio $E_{V I N E S} / \xi_{L I}$ represents the average measure of the percentage of energy dissipated by the VI NES per unit time, whereas the ratio $95 / \xi_{95 \%}$ the average percentage of energy dissipated per unit time until $95 \%$ of total energy is dissipated. It follows that the ratio $E_{V I N E S} / \xi_{L I}$ provides a measure of VI TET efficiency per unit time as long as vibro-impacts occur (i.e., for $\xi \leq \xi_{L I}$ ), and is used as a means of judging the rate (time scale) of energy dissipation (efficiency) by the VI NES only. Alternatively, the measure $95 / \xi_{95 \%}$ is used to study the overall rate of energy dissipation in the system (including the combined effects of inelastic vibro-impacts and viscous damping dissipation).

Clearly, higher values of the energy measure $E_{V I N E S}$, and/or lower values of the time measure $\xi_{95 \%}$, indicate more efficient VI TET in the system under consideration. Moreover, if there are no other sources of dissipation, higher values of the average rate $E_{V I N E S} / \xi_{L I}$ indicate high VI TET efficiency in the nonlinear regime of the damped response; i.e., in the regime where vibro-impacts are realized. However, in the presence of additional viscous damping dissipation in the $\mathrm{LO}$, the average rate $95 / \xi_{95 \%}$ provides a better indicator of the overall efficiency of TET to dissipate a significant portion of the total initial energy of the system throughout the damped motion. 

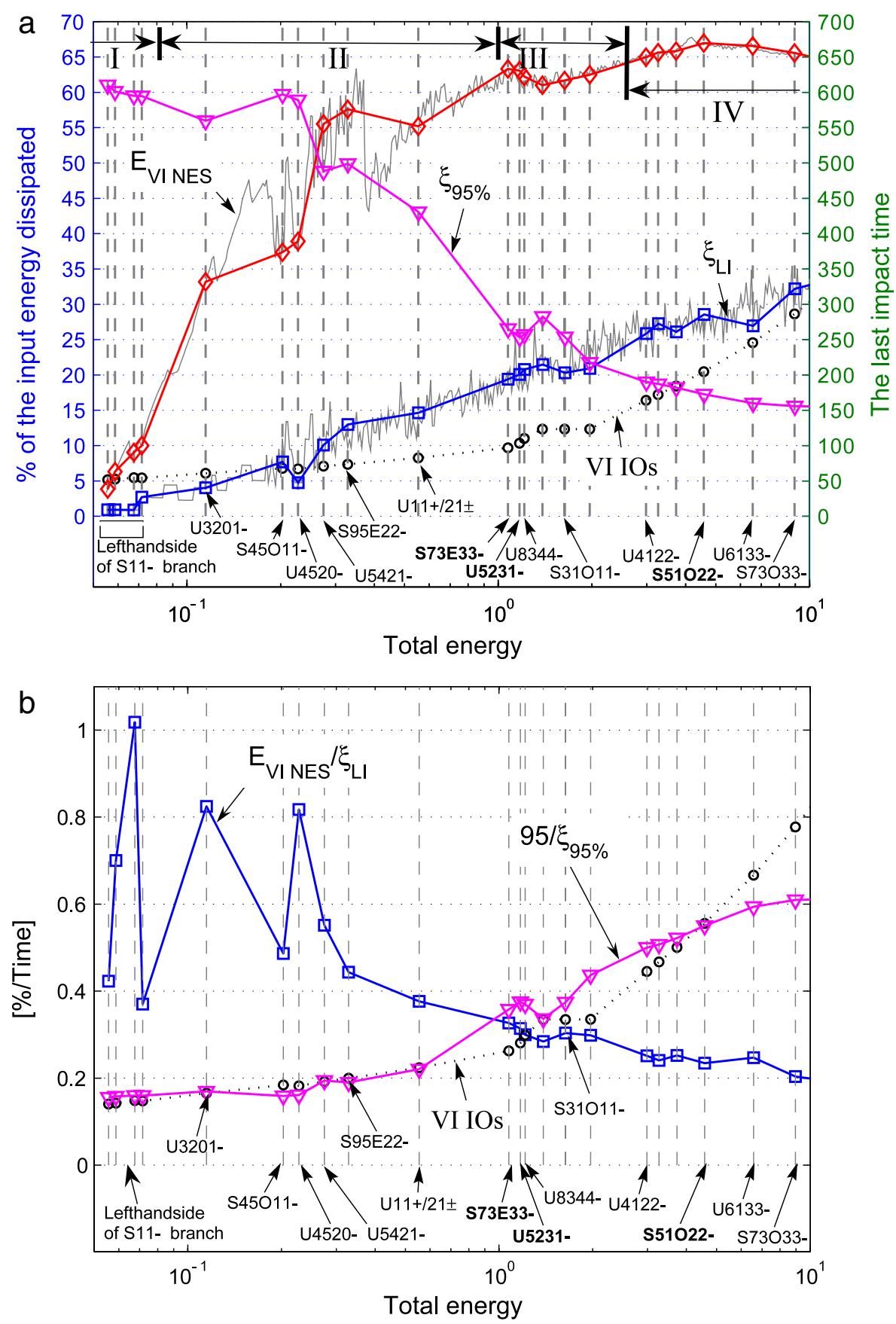

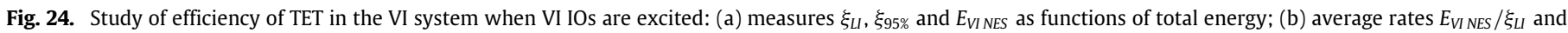
$95 / \xi_{95 \%}$ as functions of total energy $\left(\rho=0.7, \lambda=0.005 \lambda_{\text {crit }}\right)$.

In Fig. 24 we depict the measures $\xi_{L I}, \xi_{95 \%}, E_{V I N E S}$, and the average rates $E_{V I N E S} / \xi_{L I}$ and $95 / \xi_{95 \%}$ as functions of initial energy, for damped VI responses initiated on VI IOs over a wide energy range of the FEP. The system parameters used for these simulations are $\rho=0.7, \lambda=0.005 \lambda_{\text {cr }}$ and $\mu=\sigma=0.1$. As expected, with increasing energy more vibro-impacts occur, as indicated by the increase of the normalized time measure $\xi_{L I}$ with increasing energy in Fig. 24(a). Judging from the dependence of the energy measure $E_{\text {VINES }}$ on energy, we conclude that most efficient VI TET is realized when VI IOs are excited in regimes (III) and (IV) of the FEP (the highest VI TET efficiency is above $65 \%$ for this series of simulations). Moreover, VI TET in these regimes occurs at a relatively fast time scale, as indicated by the relatively small values of the normalized time measure $\xi_{95 \%}$ in the corresponding energy ranges.
Focusing now on the average rates depicted in Fig. 24(b), we deduce again that the most efficient rates of the overall energy dissipation measure $95 / \xi_{95 \%}$, are realized in regimes (III) and (IV), although the highest rates of energy dissipated by the VI NES during vibro-impacts $\left(E_{V I N E S} / \xi_{L I}\right)$ are realized in the lower energy regimes (I) and (II). We conclude that, although in these regimes there occurs strong TET from the LO to the VI NES as long as vibro-impacts last (i.e., there is more efficient energy dissipation per vibro-impact), the overall duration of vibro-impacts is small (due to the small level of overall energy), as reflected by the relatively small values of the corresponding overall TET efficiency rates $95 / \xi_{95 \%}$

In an additional series of numerical simulations we computed the previous energy dissipation measures for simulations 

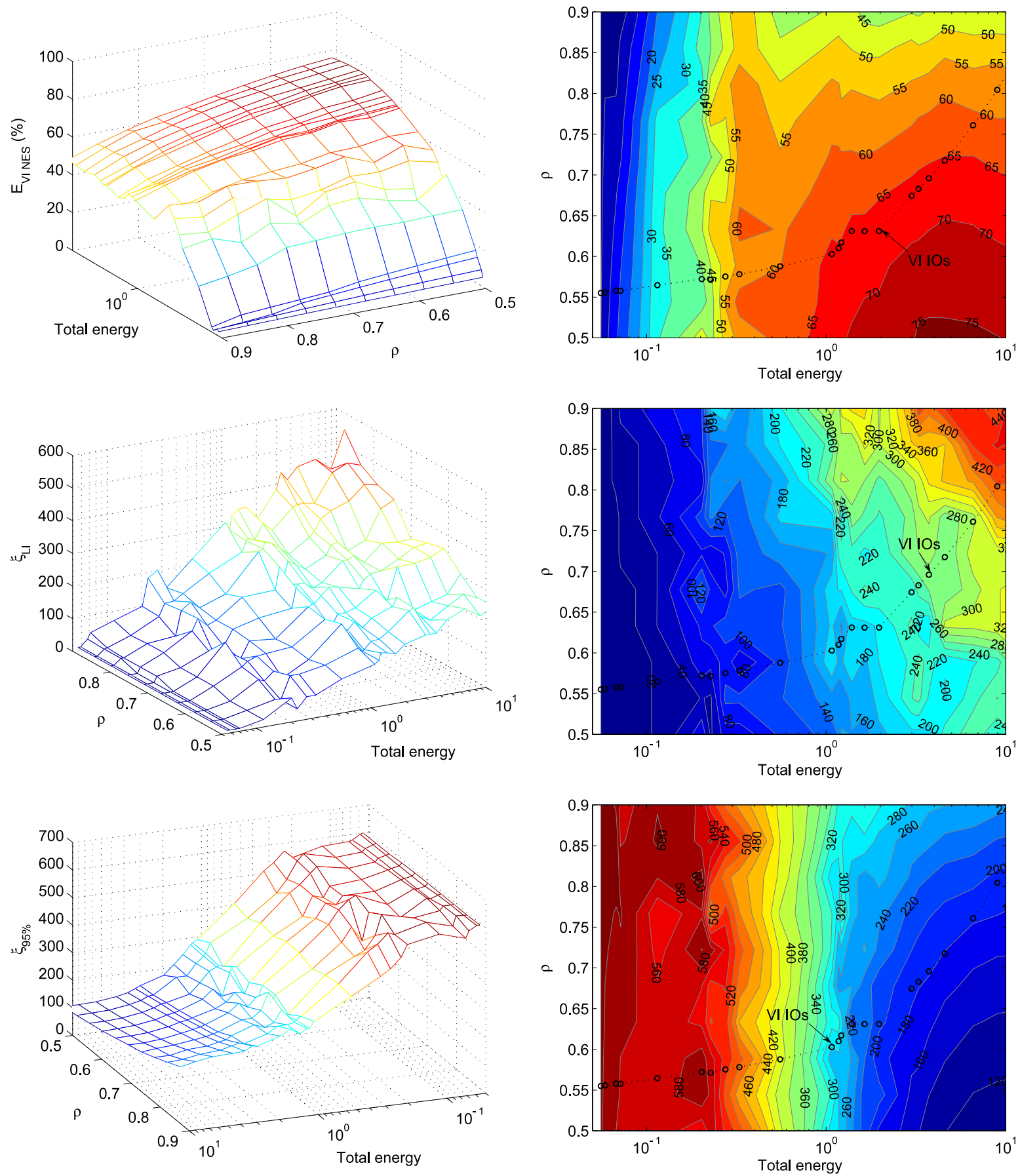

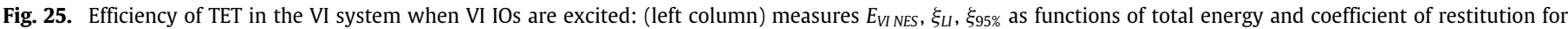
$\lambda=0.01$; (right column) corresponding projections in the plane of total energy versus coefficient of restitution.

corresponding to excitations of VI IOs at varying energies and restitution coefficients $\rho$, and fixed viscous damping coefficient $\lambda=0.005 \lambda_{c r}=0.01$. This study identified the regimes of efficient VI TET when both the energy of the excited VI IO and the coefficient of restitution of vibro-impacts are varied. The results are presented in Figs. 25 and 26, from which we conclude that the most efficient TET takes place when highly energetic VI IOs are excited in regimes (III) and (IV) of the FEP, and for smaller restitution coefficients, i.e., for highly inelastic vibro-impacts. This last result is not as obvious as it might seem from a first reading; indeed, although it is clear that the average rate $E_{V I N E S} / \xi_{L I}$ increases when the restitution coefficient increases (as this results in increased energy dissipated per vibro-impact), this does not necessarily imply that the overall TET efficiency as measured by the average rate $95 / \xi_{95 \%}$ also increases (for example, refer to the average rates depicted in Fig. 24(b)).

\section{Conclusions}

We showed that an SDOF primary linear oscillator (LO) with an attached vibro-impact nonlinear energy sink (VI NES) possesses very complicated dynamics. In the absence of energy dissipation, vibro-impacts produced by even small clearances give rise to a variety of periodic (and quasiperiodic) motions, which produce a complex topology of periodic orbits when represented in a frequency-energy plot (FEP). In the limit of zero clearance the entire FEP degenerates to two linear modes. By superimposing wavelet transform (WT) spectra of weakly damped responses 

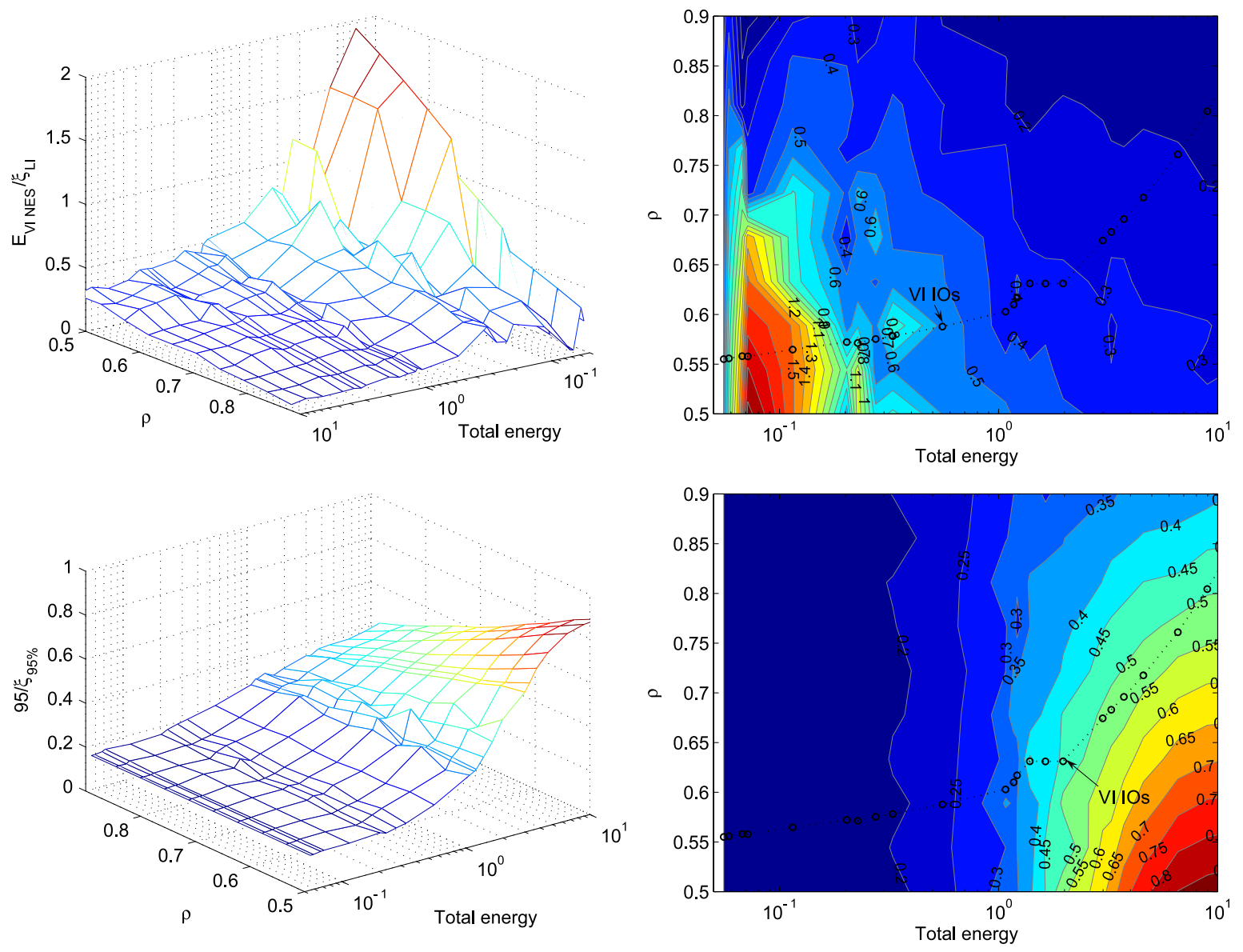

Fig. 26. Efficiency of TET in the VI system when VI IOs are excited: (left column) average rates $E_{V I N E S} / \xi_{L I}$ and $95 / \xi_{95 \%}$ as functions of total energy and coefficient of restitution for $\lambda=0.01$; (right column) corresponding projections in the plane of total energy versus coefficient of restitution.
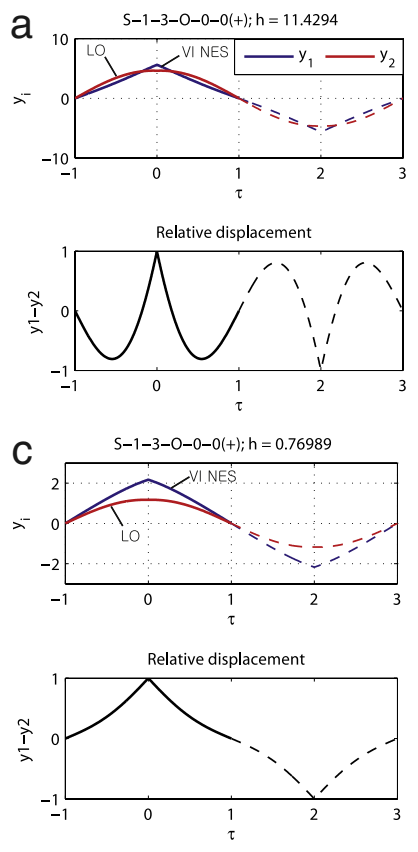
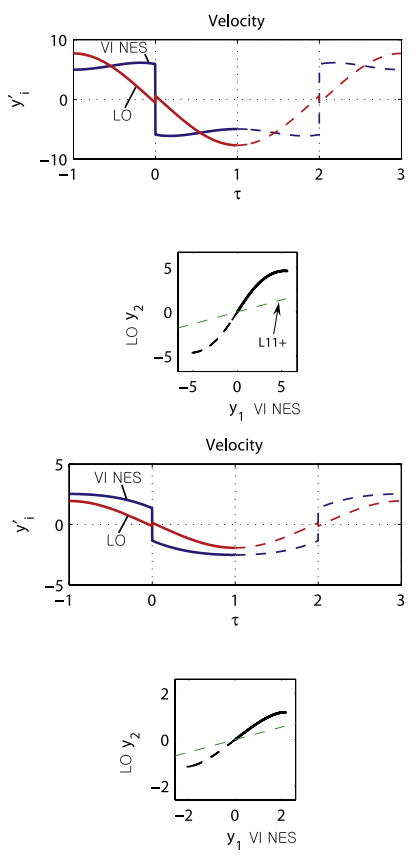
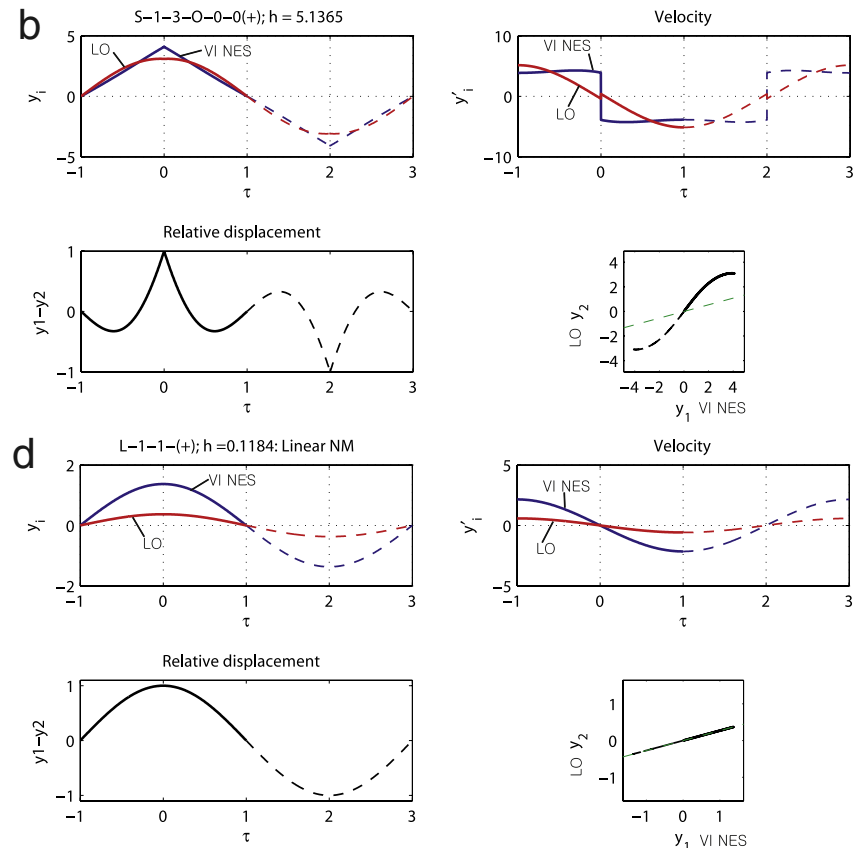
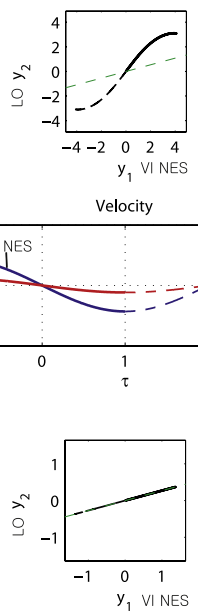

Fig. 27. S13 + branch: (a) $h=11.4294$; (b) $h=5.1365 ;$ (c) $h=0.76989 ;$ (d) $h=0.1184$.

on the Hamiltonian FEP we were able to study complicated transitions, and deduce the different mechanisms for passive targeted energy transfer (TET) from the LO to the VI NES. As in the case of smooth stiffness nonlinearity, both fundamental and subharmonic TET can be realized by the VI NES. The most efficient mechanism for TET, however, is through the excitation of highly energetic VI impulsive orbits (IOs), similar to the case of the NES with smooth (cubic) stiffness nonlinearity. In contrast to the 



Fig. 28. S11- branch: (a) $h=4.5636$; (b) $h=0.14673$; (c) $h=0.069495$; (d) $h=0.086339$.
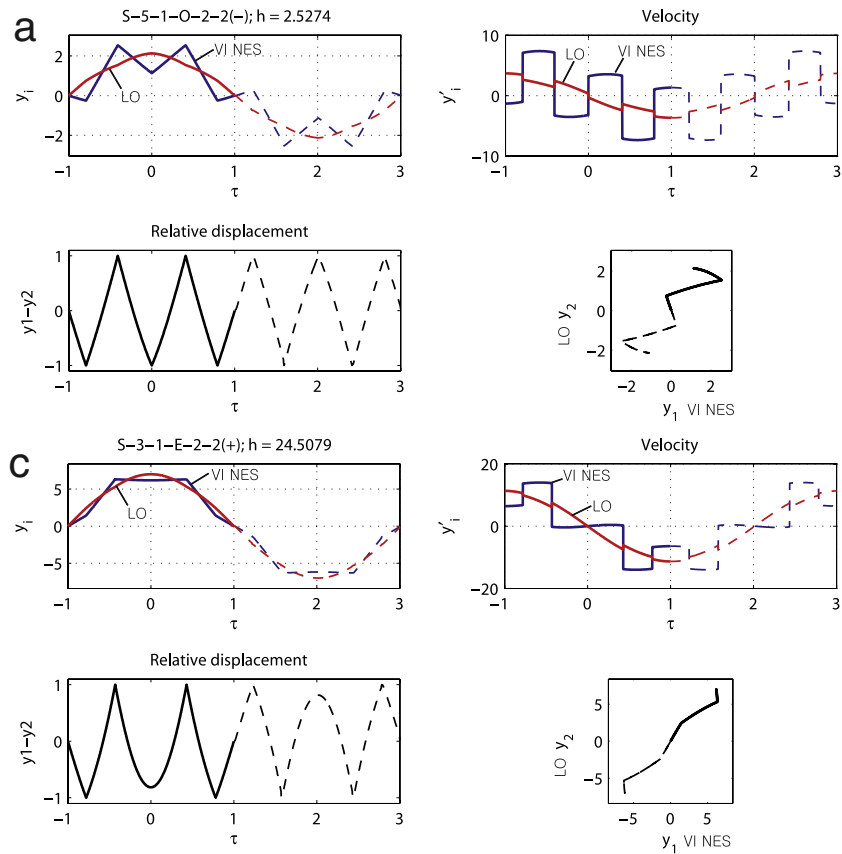

b
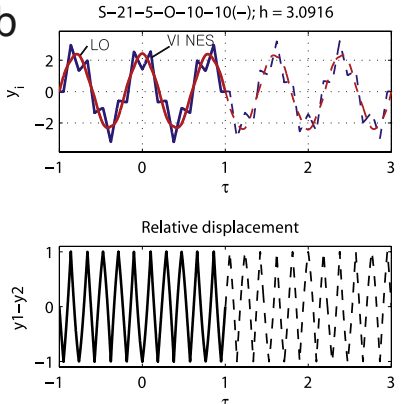

d
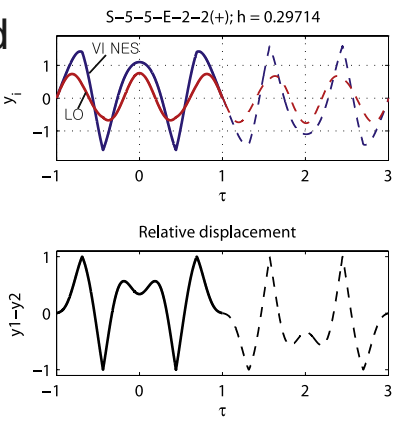
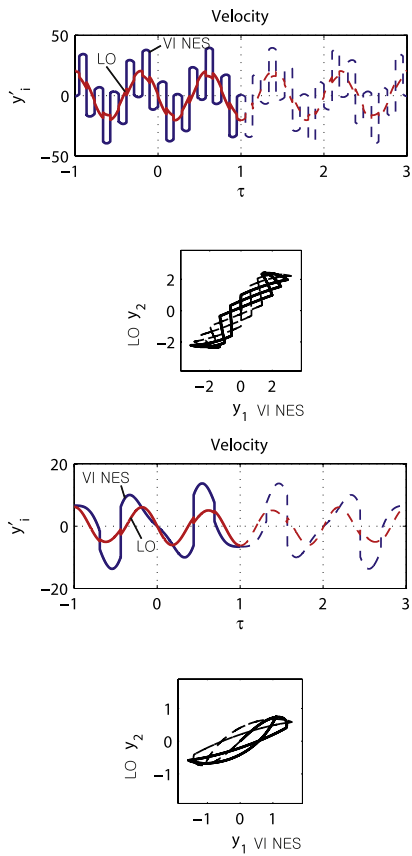

Fig. 29. Symmetric branches: (a) S51022-; (b) $S 21-5-O-10-10-$; (c) S31E22+; (d) S55E22+.

NESs with smooth essential nonlinearities, however, VI NESs are capable of passively absorbing and locally dissipating significant portions of the energy of the primary systems to which there are attached, at sufficiently fast time scales. This renders them suitable for applications like seismic mitigation, where shock elimination in the early, highly energetic regime of the motion is a critical requirement.

\section{Acknowledgments}

This work was supported in part by the US Air Force Office of Scientific Research through Grant Number FA9550-07-1-0335, and by the National Science Foundation through Grant Number CMS03-24433.

\section{Appendix. VI periodic orbits}

\section{A.1. Main backbones}

See Figs. 27 and 28.

\section{A.2. Subharmonic tongues}

See Figs. 29 and 30. 

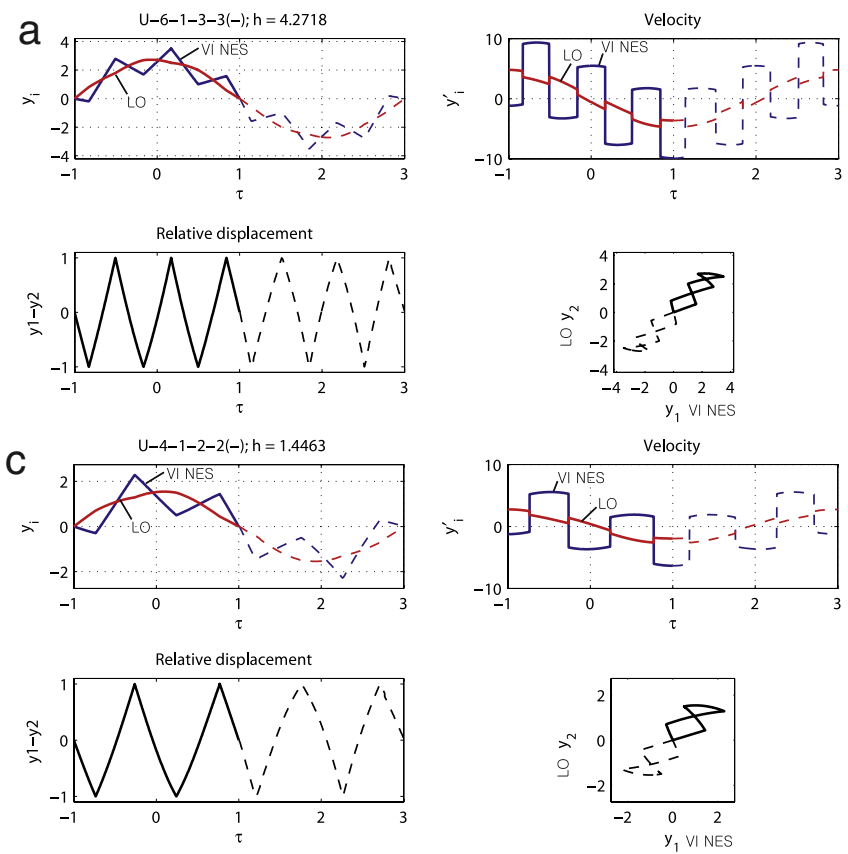
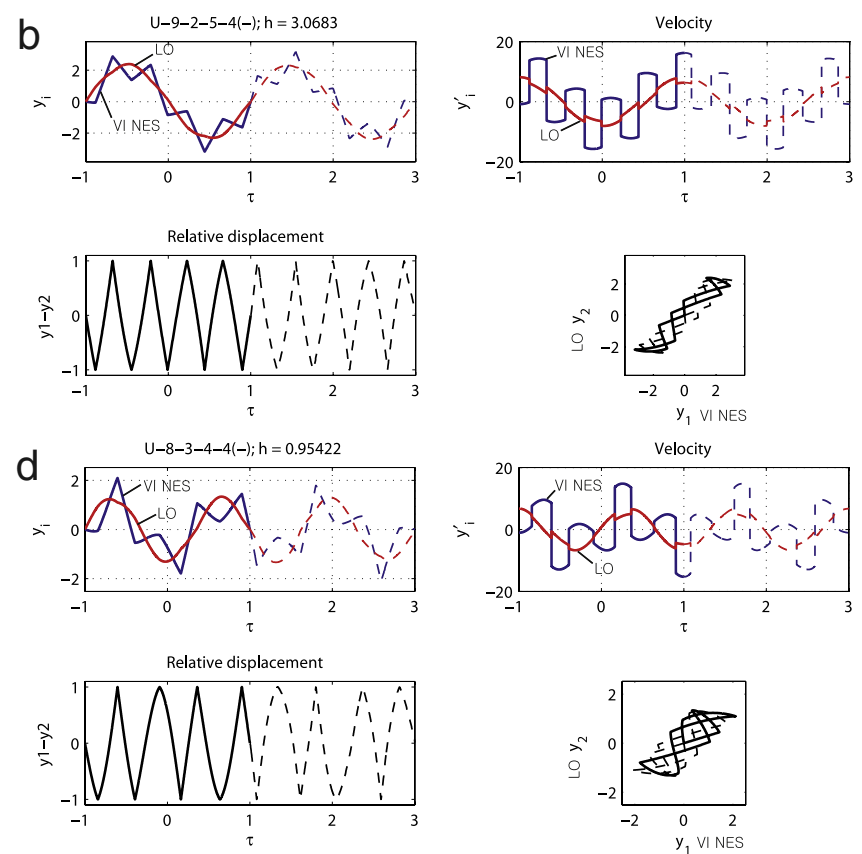

Fig. 30. Unsymmetric branches: (a) U6133-; (b) U9254-; (c) U4122-; (d) U8344-.

\section{References}

[1] O. Gendelman, L. Manevitch, A. Vakakis, R. M'Closkey, Energy pumping in coupled mechanical oscillators, Part I: Dynamics of the underlying hamiltonian systems, Trans. ASME, J. Appl. Mech. 68 (2001) 34-41.

[2] A. Vakakis, O. Gendelman, Energy pumping in coupled mechanical oscillators, Part II: Resonance capture, Trans. ASME, J. Appl. Mech. 68 (2001) 42-48.

[3] Y. Lee, A. Vakakis, L. Bergman, D.M. McFarland, G. Kerschen, F. Nucera, S. Tsakirtzis, P. Panagopoulos, Passive nonlinear targeted energy transfer (TET) and its applications to vibration absorption: A review, Proc. Inst. Mech. Eng. Part K, J. Multi-Body Dynam. 222 (2) (2008) 77-134

[4] A. Vakakis, O. Gendelman, L. Bergman, D.M. McFarland, G. Kerschen, Y. Lee Passive Nonlinear Targeted Energy Transfer in Mechanical and Structural Systems: I and II, Springer-Verlag, Berlin, New York, 2008

[5] F. Nucera, A. Vakakis, D.M. McFarland, L. Bergman, G. Kerschen, Targeted energy transfers in vibro-impact oscillators for seismic mitigation, Nonlinear Dynam. 50 (3) (2007) 651-677.

[6] F. Nucera, F.L. Iacono, D.M. McFarland, L. Bergman, A. Vakakis, Application of broadband nonlinear targeted energy transfers for seismic mitigation of a shear frame: Experimental results, J. Sound Vib. 313 (1-2) (2008) 57-76.

[7] F. Nucera, D.M. McFarland, L. Bergman, A. Vakakis, Application of broadband nonlinear targeted energy transfers for seismic mitigation of a shear frame: Computational results, J. Sound Vib. (in press).

[8] F. Georgiadis, A. Vakakis, D.M. McFarland, L. Bergman, Shock isolation through passive energy pumping caused by nonsmooth nonlinearities, Internat. J. Bifur. Chaos 15 (6) (2005) 1989-2001.

[9] F. Georgiades, Nonlinear localization and targeted energy transfer phenomena in vibrating systems with smooth and non-smooth stiffness nonlinearities, Ph.D. Thesis, National Technical University of Athens, Athens, Greece, 2006.

[10] I. Karayannis, A. Vakakis, F. Georgiades, Vibro-impact attachments as shock absorbers, Proc. Inst. Mech. Eng. Part C: J. Mech. Eng. Sci. 222 (2008) 1899-1908.

[11] S. Shaw, R. Rand, The transition to chaos in a simple mechanical system, Internat. J. Non-Linear Mech. 24 (1989) 41-56.

[12] V. Babitsky, Theory of Vibro-impact Systems, Springer Verlag, Berlin, New York, 1998.

[13] B. Brogliato, Non-smooth Mechanics, Springer Verlag, Berlin, New York, 1999

$14]$ S. Kryzhevich, V. Pliss, Chaotic modes of oscillation of a vibro-impact system J. Appl. Math. Mech. (PMM) 69 (2005) 13-26.

[15] P. Thota, X. Zhao, H. Dankowicz, Co-dimension-two grazing bifurcations in single-degree-of-freedom impact oscillators, J. Comput. Nonlinear Dynam. 1 (2006) 328-335.

[16] P. Thota, H. Dankowicz, Continuous and discontinuous grazing bifurcations in impacting oscillators, Physica D 214 (2006) 187-197.

[17] S. Masri, T. Caughey, On the stability of the impact damper, Trans. ASME, J. Appl. Mech. 33 (3) (1966) 586-592.

[18] S. Masri, A. Ibrahim, Response of the impact damper to nonstationary random excitation, J. Acoust. Soc. Am. 53 (1) (1973) 200-211.

[19] A. Filippov, Differential Equations with Discontinuous Righthand Sides, Kluwer Academic Publishers, 1988.

[20] F. Pfeiffer, C. Glocker, Contacts in multibody systems, J. Appl. Math. Mech. 64 (5) (2000) 773-782.
[21] B. Blazejczyk-Okolewska, Analysis of an impact damper of vibration, Chaos Solitons Fractals 12 (2001) 1983-1988.

[22] R. Leine, H. Nijmeijer, Dynamics and Bifurcations in Non-Smooth Mechanical Systems, Springer-Verlag, Berlin, New York, 2004.

[23] F. Peterka, B. Blazejczyk-Okolewska, Some aspects of the dynamical behavior of the impact damper, J. Vib. Control 11 (2005) 459-479.

[24] J.Q. Sun, A. Luo, Bifurcation and Chaos in Complex Systems, Elsevier, 2006

[25] S. Shaw, P. Holmes, A periodically forced piecewise linear oscillator, J. Sound Vib. 90 (1) (1983) 129-155.

[26] J. Shaw, S. Shaw, The onset of chaos in a two-dof impacting system, Trans. ASME, J. Appl. Mech. 56 (1989) 168-174.

[27] S. Shaw, The dynamics of a harmonically excited system having rigid amplitude constraints. I: Subharmonic motions and local bifurcations. II: Chaotic motions and global bifurcations, Trans. ASME, J. Appl. Mech. 52 (2) (1985) 453-464.

[28] I. Gorelyshev, A. Neishtadt, On the adiabatic perturbation theory for subsystems with impacts, J. Appl. Math. Mech. 70 (2006) 4-17.

[29] Y. Mikhlin, A. Vakakis, G. Salenger, Direct and inverse problems encountered in vibro-impact oscillations of a discrete system, J. Sound Vib. 216 (1998) $227-250$.

[30] R. Leine, D.V. Campen, B. van de Vrande, Bifurcations in nonlinear discontinuous systems, Nonlinear Dynam. 23 (2000) 105-164

[31] K. Czolczynski, T. Kapitaniak, On the existence of a stable periodic solution of two impacting oscillators with damping, Internat. J. Bifur. Chaos 14(11)(2004) 3931-3947.

[32] G. Wen, J. Xie, D. Xu, Onset of degenerate hopf bifurcation of a vibro-impact oscillator, Trans. ASME, J. Appl. Mech. 71 (2004) 579-581.

[33] M. Dupac, D. Marghitu, Nonlinear dynamics of a flexible mechanism with impact, J. Sound Vib. 289 (2006) 952-966.

[34] R. Leine, Bifurcations of equilibria in non-smooth continuous systems, Physica D 223 (2006) 121-137.

[35] W. Lin, Q. Ni, Y. Huang, Bifurcations and chaos in a forced cantilever system with impacts, J. Sound Vib. 296 (2006) 1068-1078.

[36] C. Halse, R. Wilson, M. di Bernardo, M. Homer, Coexisting solutions and bifurcations in mechanical oscillators with backlash, J. Sound Vib. 305 (2007) $854-885$.

[37] G. Luo, Y. Zhang, Y. Chu, J. Zhang, Co-dimension-two bifurcations of fixed points in a class of vibratory systems with symmetrical rigid stops, Nonlinear Anal. 8 (2007) 1272-1292.

[38] V. Zhuravlev, A method of analyzing vibro-impact systems using special functions, Izv. Akad. Nauk. SSSR, MTT 2 (1976) 30-34.

[39] V. Zhuravlev, Investigation of some vibro-impact systems by the method of non-smooth transformations, Izv. Akad. Nauk. SSSR, MTT 6 (1977) 24-28.

[40] M. Azeez, A. Vakakis, L. Manevitch, Exact solutions of the problem of the vibroimpact oscillations of a discrete system with two degrees-of-freedom, J. Appl. Math. Mech. (PMM) 63 (4) (1999) 527-530.

[41] V. Pilipchuk, The calculation of strongly nonlinear systems close to vibrationimpact systems, J. Appl. Math. Mech. (PMM) 49 (1985) 572-578.

[42] R. Pinnington, Energy dissipation prediction in a line of colliding oscillators, J. Sound Vib. 268 (2003) 361-384. 
[43] S. Salapaka, M. Dahleh, I. Mezic, On the dynamics of a harmonic oscillator undergoing impacts with a vibrating platform, Nonlinear Dynam. 24 (2001) 333-358.

[44] D. Quinn, The dynamics of two parametrically excited pendula with impacts, Internat. J. Bifur. Chaos 15 (6) (2005) 1975-1988.

[45] K. Li, A. Darby, Experiments on the effect of an impact damper on a MDOF system, J. Vib. Control 12 (5) (2006) 445-464.

[46] K. Murphy, T. Morrison, Grazing instabilities and post-bifurcation behavior in an impacting string, J. Acoust. Soc. Am. 111 (2) (2002) 884-892.

[47] B. Hu, W. Schiehlen, Multi-time scale simulation for impact systems: From wave propagation to rigid-body motion, Arch. Appl. Mech. (Ingenieur Archiv) 72 (11) (2003) 885-898.

[48] R. Sampaio, C. Soize, On measures of nonlinearity effects for uncertain dynamical systems - Applications to a vibro-impact system, J. Sound Vib. 303 (2007) 659-674.

[49] M. Azeez, A. Vakakis, Proper orthogonal decomposition (POD) of a class of vibro-impact oscillations, J. Sound Vib. 240 (5) (2001) 859-889.

[50] Y. Vedenova, L. Manevitch, V. Pilipchuk, The normal vibrations of a string with concentrated masses on nonlinearly elastic supports, Prik. Mat. Mech. 49 (2) (1985) 203-211.

[51] O.V. Gendelman, Modeling of inelastic impacts with the help of smoothfunctions, Chaos Solitons Fractals 28 (2) (2006) 522-526.

[52] D. Meimukhin, O. Gendelman, Response regimes of integrable damped strongly nonlinear oscillator under impact periodic forcing, Chaos Solitons Fractals 32 (2) (2007) 405-414.

[53] R. Nayeri, S. Masri, J. Caffrey, Studies of the performance of multi-unit impact dampers under stochastic excitation, J. Vib. Acoust. 129 (2007) 239-251.

[54] S. Namachchivaya, J. Park, Stochastic dynamics of impact oscillators, Trans. ASME, J. Appl. Mech. 72 (2005) 862-870.

[55] D. Wagg, A note on coefficient of restitution models including the effects of impact induced vibration, J. Sound Vib. 300 (2007) 1071-1078.

[56] S. Shaw, C. Pierre, The dynamic response of tuned impact absorbers for rotating flexible structures, J. Comput. Nonlinear Dynam. 1 (2006) 13-24.

[57] Y. Lee, G. Kerschen, A. Vakakis, P. Panagopoulos, L. Bergman, D.M. McFarland, Complicated dynamics of a linear oscillator with a light, essentially nonlinear attachment, Physica D 204 (1-2) (2005) 41-69.

[58] G. Kerschen, Y. Lee, A. Vakakis, D.M. McFarland, L. Bergman, Irreversible passive energy transfer in coupled oscillators with essential nonlinearity, SIAM J. Appl. Math. 66 (2) (2006) 648-679.
[59] V. Pilipchuk, A. Vakakis, M. Azeez, Study of a class of subharmonic motions using a non-smooth temporal transformation (NSTT), Physica D 100 (1997) 145-164.

[60] A. Vakakis, Analysis and identification of linear and nonlinear normal modes in vibrating systems, Ph.D. Thesis, California Institute of Technology, 1991.

[61] S. Roberts, J. Shipman, Two-Point Boundary Value Problems: Shooting Methods, American Elsevier Publishing Company, Inc., New York, 1972.

[62] S.N. Ha, A nonlinear shooting method for two-point boundary value problems, Comput. Math. Appl. 42 (10-11) (2001) 1411-1420.

[63] B.S. Attili, M.I. Syam, Efficient shooting method for solving two point boundary value problems, Chaos Solitons Fractals 35 (5) (2008) 895-903.

[64] L. Manevitch, Y. Mikhlin, V. Pilipchuk, The Method of Normal Modes for Essentially Nonlinear Systems, Nauka, Moscow, 1989.

[65] V. Pilipchuk, Analytical study of vibrating systems with strong non-linearities by employing saw-tooth time transformation, J. Sound Vib. 192 (1996) 43-64.

[66] A. Vakakis, L. Manevitch, Y. Mikhlin, V. Pilipchuk, A. Zevin, Normal Modes and Localization in Nonlinear Systems, John Wiley \& Sons, Inc., 1996.

[67] G. Salenger, A. Vakakis, Discreteness effects in the forced dynamics of a string on a periodic array of non-linear supports, Internat. J. Non-Linear Mech. 33 (1998) 659-673.

[68] G. Kerschen, A. Vakakis, Y. Lee, D.M. Mcfarland, J. Kowtko, L. Bergman, Energy transfers in a system of two coupled oscillators with essential nonlinearity: $1: 1$ resonance manifold and transient bridging orbits, Nonlinear Dynam. 42 (3) (2005) 283-303.

[69] G. Kerschen, O. Gendelman, A.F. Vakakis, L A Bergman, D.M. McFarland, Impulsive periodic and quasi-periodic orbits of coupled oscillators with essential stiffness nonlinearity, Commun. Nonlinear Sci. Numer. Simul. 13 (5) (2008) 959-978.

[70] N. Huang, Z. Shen, S. Long, M. Wu, H. Shih, Q. Zheng, N.-C. Yen, C. Tung, H. Liu, The empirical mode decomposition and the Hilbert spectrum for nonlinear and non-stationary time series analysis, Proc. Roy. Soc. London Ser. A. Math. Phys. Sci. 454 (1998) 903-995.

[71] G. Kerschen, A. Vakakis, Y. Lee, D.M. McFarland, L. Bergman, Toward a fundamental understanding of the Hilbert-Huang transform in nonlinear structural dynamics, J. Vib. Control 14 (1-2) (2008) 77-105.

[72] G. Rilling, P. Flandrin, P. Gonoalvès, On empirical mode decomposition and its algorithms, in: IEEE-Eurasip Workshop on Nonlinear Signal and Image Processing, Grado, Italy, June 2003. 